Article

\title{
In Silico Insights into HIV-1 Vpu-Tetherin Interactions and Its Mutational Counterparts
}

\author{
Patil Sneha ${ }^{1,2}$, Urmi Shah ${ }^{1}$ and Seetharaman Balaji ${ }^{3, * \mathbb{C}}$ \\ 1 School of Biotechnology and Bioinformatics, D Y Patil Deemed to be University, Plot 50, Sector 15, CBD \\ Belapur, Navi Mumbai, Maharashtra 400614, India; sneha.patil@dypatil.edu (P.S.); \\ urmisha.bt16@dypatil.edu (U.S.) \\ 2 Research and Development, Bharathiar University, Coimbatore, Tamil Nadu 641046, India \\ 3 Department of Biotechnology, Manipal Institute of Technology, Manipal Academy of Higher Education, \\ Manipal, Karnataka 576104, India \\ * Correspondence: s.balaji@manipal.edu
}

Received: 21 May 2019; Accepted: 19 June 2019; Published: 22 June 2019

check for updates

\begin{abstract}
Tetherin, an interferon-induced host protein encoded by the bone marrow stromal antigen 2 (BST2/CD317/HM1.24) gene, is involved in obstructing the release of many retroviruses and other enveloped viruses by cross-linking the budding virus particles to the cell surface. This activity is antagonized in the case of human immunodeficiency virus (HIV)-1 wherein its accessory protein Viral Protein $\mathrm{U}(\mathrm{Vpu})$ interacts with tetherin, causing its downregulation from the cell surface. Vpu and tetherin connect through their transmembrane (TM) domains, culminating into events leading to tetherin degradation by recruitment of $\beta-\operatorname{TrCP} 2$. However, mutations in the TM domains of both proteins are reported to act as a resistance mechanism to Vpu countermeasure impacting tetherin's sensitivity towards Vpu but retaining its antiviral activity. Our study illustrates the binding aspects of blood-derived, brain-derived, and consensus HIV-1 Vpu with tetherin through protein-protein docking. The analysis of the bound complexes confirms the blood-derived Vpu-tetherin complex to have the best binding affinity as compared to other two. The mutations in tetherin and Vpu are devised computationally and are subjected to protein-protein interactions. The complexes are tested for their binding affinities, residue connections, hydrophobic forces, and, finally, the effect of mutation on their interactions. The single point mutations in tetherin at positions L23Y, L24T, and P40T, and triple mutations at $\{\mathrm{L} 22 \mathrm{~S}, \mathrm{~F} 44 \mathrm{Y}, \mathrm{L} 37 \mathrm{I}\}$ and $\{\mathrm{L} 23 \mathrm{~T}, \mathrm{~L} 37 \mathrm{~T}, \mathrm{~T} 45 \mathrm{I}\}$, while single point mutations in Vpu at positions $\mathrm{A} 19 \mathrm{H}$ and $\mathrm{W} 23 \mathrm{Y}$ and triplet of mutations at $\{\mathrm{V} 10 \mathrm{~K}, \mathrm{~A} 11 \mathrm{~L}, \mathrm{~A} 19 \mathrm{~T}\},\{\mathrm{V} 14 \mathrm{~T}, \mathrm{I} 18 \mathrm{~T}, \mathrm{I} 26 \mathrm{~S}\}$, and $\{\mathrm{A} 11 \mathrm{~T}, \mathrm{~V} 14 \mathrm{~L}, \mathrm{~A} 15 \mathrm{~T}\}$ have revealed no polar contacts with minimal hydrophobic interactions between $\mathrm{Vpu}$ and tetherin, resulting in reduced binding affinity. Additionally, we have explored the aggregation potential of tetherin and its association with the brain-derived Vpu protein. This work is a possible step toward an understanding of Vpu-tetherin interactions.
\end{abstract}

Keywords: HIV-1 Vpu; tetherin; transmembrane interactions; aggregation potential

\section{Introduction}

Tetherin, a protein encoded by the BST2 gene, also known as bone marrow stromal antigen 2/ CD317/HM1.24, is an integral membrane protein involved in the interferon dependent antiviral response pathway [1]. The antiviral activity of tetherin is bestowed upon by its unique topology that blocks the budding viruses and prevents them from leaving the cell [2]. Its N-terminal region is in the cytoplasm with a membrane spanning the helical domain (transmembrane (TM) domain) and an alpha helical coiled coil ectodomain with a glycosyl-phosphatidylinositol modified anchor at C-terminal [3,4]. Tetherin serves as a potent inhibitor of enveloped viruses, like human immunodeficiency virus (HIV), 
by its tethering phenomena, eventually leading to lysosomal degradation of tethered viral particles [5]. However, HIV-1 establishes a cogent mechanism against this defensive strategy of tetherin by expressing its exclusive accessory protein Viral Protein U (Vpu) [6]. Vpu, an 81-amino acid oligomeric protein, consists of an N-terminal TM domain associated with release of viral particles and a C-terminal domain involved in viral receptors-CD4 degradation [7]. The enhancement of viral budding and release by $\mathrm{Vpu}$ is attributed to the dislocation of tetherin from the cell surface causing its internalization and possible lysosomal degradation eventually overcoming its restriction [4]. The phosphorylated conserved serine residues S52 and S56 in the cytoplasmic tail of Vpu are found to be efficient in tetherin degradation [8]. These residues are recognized by an F-box-containing ubiquitin ligase subunit, the beta-transducin repeat-containing protein-2 ( $\beta$-TrCP-2). Vpu recruits the multi-subunit SCF- $\beta$-TrCP E3 ubiquitin ligase complex that causes ubiquitination and degradation of BST-2 [8-10]. Thus, its downregulation is partially controlled by $\beta-\mathrm{TrCP}$, which is also linked to $\mathrm{Vpu}$ induced proteosomal CD4 degradation. This is the result of the TM interactions between Vpu and tetherin that aid in bringing the cytoplasmic tail of Vpu in the vicinity of tetherin, leading to its displacement [9].

The binding of Vpu to tetherin at their TM domains is a helix-helix interaction that is a crucial for antagonizing antiviral activity [11,12]. The amino acids residing in and around the Vpu-tetherin TM domains present vital aspects in understanding the helix-helix association between the two and determining the susceptibility of tetherin to Vpu. Studies have revealed that the mutations in the TM regions of Vpu or tetherin have rendered tetherin resistant to Vpu antagonism [13-15]. A study has shown that a single point mutation in tetherin T45I was successful in rendering tetherin resistant to Vpu-mediated depletion [16], while numerous other studies dealing with identification of interacting points between the two proteins have put forth positions crucial for binding and mutations in them affecting their binding potential [13-19]. These studies have specified Vpu residues A14, A18, and W22, as well as tetherin residues L22, L23, G25, I26, V30, I33, I34, I36, L37, L41, and T45, to be participating in the interaction [13-19].

In this study, the in silico interactions between HIV-1 Vpu and tetherin were performed. As compartmentalization of HIV-1 in different organs, especially in the central nervous system (CNS), is likely to generate distinct Vpu isolates with varying residues [20-22], the Vpu sequences used were isolates of two distinct compartments, the brain and blood. A consensus Vpu sequence was also used in an attempt to highlight the differences in their binding potential. On this basis, the selected amino acid positions of tetherin and blood-derived HIV-1 Vpu were considered for mutational study. The differences in their binding affinities and the interacting residues have been charted out for selected mutations along with the aggregating potential of tetherin.

\section{Materials and Methods}

\subsection{Sequence and Structure Retrieval}

The representative sequences of blood- and brain-derived HIV-1 Vpu proteins were retrieved from UniprotKB (http://www.uniprot.org/) with accession numbers P35966 and P12516, respectively. These sequences are part of around 50 blood- and 39 brain-derived HIV-1 Vpu protein sequences that were collected and analyzed for their sequence specific variations from different geographical locations [22]. The structure of brain-derived HIV-1 Vpu (P12516) used in the current study has been predicted and validated in our previous work on amyloidogenicity study of HIV-1 Vpu [23]. To analyze the interaction between HIV-1 Vpu and tetherin, the structure of tetherin TM domain (protein data bank (PDB) ID: 2LK9) was retrieved from the protein data bank (http://www.rcsb.org/) [24].

\subsection{Multiple Sequence Alignment and Generation of Consensus Vpu Sequence}

The multiple sequence alignment of geographically divergent 89 blood- and brain-derived HIV-1 Vpu sequences was carried out in Clustal Omega (https://www.ebi.ac.uk/Tools/msa/clustalo/). Clustal Omega applies seeded guide trees and Hidden Markov Model (HMM) profile-profile methods for 
ensuring an optimal alignment between the given sequences [25]. The consensus sequence was obtained from an Emboss explorer, a server for creating consensus sequence from multiple alignment (http://www.bioinformatics.nl/cgi-bin/emboss/cons) [26]. The consensus was deduced with a default plurality value taken as half the total weight of all the sequences in the alignment. The variations in blood and brain Vpu residue positions from consensus $\mathrm{Vpu}$ sequence generated are represented in Figure 1. The geographically and compartmentally distinct HIV-1 Vpu proteins were compiled together in a consensus sequence to form a representative of a complete Vpu blood and brain dataset and further aid in understanding the interactions between Vpu-tetherin complexes.

\begin{tabular}{|c|c|}
\hline VPU_consensus & MOpLQILAIVALVVAaIIAIVVUSIVFIEYRKILRQRKIDRLIDRIRERAEDSGNESEGD \\
\hline VPU_blood & LQSLQVLAIVALVWATIIAIVWTTIVFIEYRKILRQRKIDRLINRITERAEDSGNESDGD \\
\hline VPU_brain & 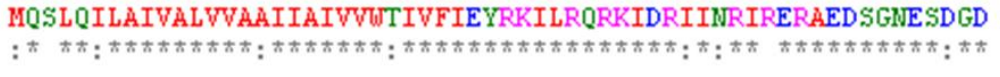 \\
\hline VPU_consensus & QEELSALVVEMGHHAPWDVDDL \\
\hline VPU_blood & QEELSALVE-RGHLAPWDVDDL \\
\hline VPU_brain & 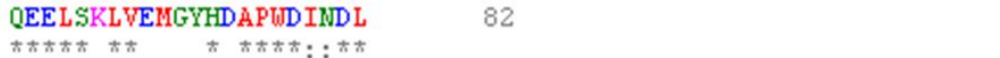 \\
\hline
\end{tabular}

Figure 1. Representation of identity $\left(^{*}\right)$ and conserved substitutions (:) between human immunodeficiency virus (HIV)-1 Viral Protein U (Vpu) sequences from blood and brain isolates and the consensus Vpu sequence.

\subsection{Protein Structure Modeling and Validation}

The tertiary structures of representative blood-derived Vpu and consensus Vpu sequences were modeled based on homology. A BLAST similarity search [27] was performed against the PDB database with a Blosum62 substitution matrix and default parameters to select a template with a good alignment score and maximum query coverage. The template with PDB ID: 2N28, having an identity score of $75 \%$ and query coverage of $72 \%$ for blood-derived Vpu sequence and identity score of $82 \%$ and query coverage of $73 \%$ for consensus Vpu sequence, was selected for homology modeling. The structure of the selected template (PDB ID: 2N28) determined using the Nuclear Magnetic Resonance (NMR) method was retrieved from PDB (http://www.rcsb.org/) [24]. The Vpu structures were modeled using the SWISS-MODEL server, an automated protein homology-modeling server (https://swissmodel.expasy.org/) [28]. Energy minimization was done using the inbuilt GROMOS96 force field. The overall model quality estimation and validation of blood- and brain-derived Vpu structures was done in protein structure analysis (ProSA-web) tool (https://prosa.services.came.sbg. ac.at/prosa.php) and the quality was assessed based on Ramachandran's plot using PROCHECK (http://servicesn.mbi.ucla.edu/PROCHECK/) [29,30].

\subsection{Protein-Protein Interaction}

In order to comprehend the binding profile of Vpu with tetherin, the wild-type representative blood- and brain-derived Vpu structures and consensus Vpu structure were subjected for interactions with the TM tetherin structure (ID: 2LK9). This was performed using Hex version 8.0.0, an interactive molecular graphics application for performing molecular interactions. Hex enables modeling of each molecule, employing 3D extensions of real orthogonal spherical polar built functions encrypting both surface shape and electrostatic charge and potential distributions. Hex illustrates the surface profiles of proteins applying a two-term surface skin and Van der Waals steric density model. With the use of appropriate scaling factors, the docking score is inferred as a minimized interaction energy [31,32]. Docking was performed using a reference complex describing the anti-parallel orientation of $\mathrm{Vpu}$ and tetherin, as reported in the available literature [11]. The set of interacting complexes were then submitted to PROtein binDIng enerGY prediction (PRODIGY) web server for prediction of binding affinity in protein-protein complexes based on intermolecular interaction contacts and characteristics 
resulting from non-interface surface. (https://nestor.science.uu.nl/prodigy/) [33,34]. The complexes were visualized and analyzed in PyMOL v 2.2.3 [35] and Swiss PDB Viewer (SPDBV) version 4.10 or "Deep view" [36]. The interacting residues between Vpu and tetherin were scrutinized in a proteins interaction calculator (PIC) server that evaluates various hydrophobic and ionic interactions, hydrogen bonds, disulfide bridges, and aromatics interactions between the proteins comprising the complexes (http://pic.mbu.iisc.ernet.in/) [37].

\subsection{Mutational Study}

The possible mutations were analyzed in I-Mutant 3.0 [38] and Sorting Intolerant From Tolerant, SIFT [39]. I-Mutant3.0 is a Support Vector Machine-based web server for predicting the effect of single point mutations on protein stability taking protein sequence or structure as input. SIFT predicts whether an amino acid substitution disturbs the protein function. SIFT prediction is grounded on the degree of conservation of amino acid residues in sequence alignments resulting from closely related sequences, composed through PSI-BLAST. The mutations in the protein structures are then performed in Chimera v 1.11.2, a protein visualization and analysis tool [40]. The structure editing menu option in Chimera aids in selection of best probability rotamer for the defined mutation at the desired position. The following amino acids in tetherin, such as L22, L23, L24, G25, I26, L29, V30, I33, I34, I36, L37, V39, P40, L41, F44, and T45, were selected for mutations (Table 1). Similarly, the following amino acids of blood-derived Vpu, such as S3, Q5, L7, A8, A11, V14, A15, I17, A19, W23, I25, F27, R31, and K32, were selected for mutations (Table 2). Mutations were introduced in the blood-derived $\mathrm{Vpu}$, as it had the best binding affinity of $-5.0 \mathrm{kcal} / \mathrm{mol}(\Delta \mathrm{G})$ in $\mathrm{Vpu}$-tetherin docked complexes in comparison with the brain-derived and consensus Vpu structures. Each selected position in tetherin and Vpu were mutated with synonymous, as well as non-synonymous, substitutions and tested for tolerance and protein stability in I-Mutant 3.0 and SIFT (Tables 1 and 2). The reliability index (RI) is computed only when the sign of the stability change is predicted and DDG $(\mathrm{kJ} / \mathrm{mol})$ indicates the free energy change upon mutation. SIFT score calculates the effect of amino acid substitution on protein function ranging from 0.0 (deleterious) to 1.0 (tolerated).

Table 1. Mutation Analysis of tetherin in I-Mutant 3.0 and Sorting Intolerant From Tolerant (SIFT). RI = reliability index.

\begin{tabular}{|c|c|c|c|c|c|c|c|}
\hline Position & Substitution & Characteristic & $\begin{array}{c}\text { SIFT } \\
\text { Tolerated }\end{array}$ & $\begin{array}{l}\text { SIFT } \\
\text { Score }\end{array}$ & $\begin{array}{l}\text { I-Mutant } \\
\text { Stability }\end{array}$ & RI & $\begin{array}{c}\text { DDG } \\
(\mathrm{kJ} / \mathrm{mol})\end{array}$ \\
\hline \multirow{5}{*}{22} & $\mathrm{~L} \rightarrow \mathrm{S}$ & Uncharged Polar & No & 0.01 & Decrease & 8 & -1.31 \\
\hline & $\mathrm{L} \rightarrow \mathrm{M}$ & Nonpolar & Yes & 0.07 & Decrease & 5 & -0.85 \\
\hline & $\mathrm{L} \rightarrow \mathrm{K}$ & Basic & No & 0.01 & Decrease & 8 & -1.36 \\
\hline & $\mathrm{L} \rightarrow \mathrm{F}$ & Nonpolar & Yes & 0.05 & Decrease & 7 & -0.89 \\
\hline & $\mathrm{L} \rightarrow \mathrm{Y}$ & Uncharged Polar & No & 0.01 & Decrease & 2 & -1.07 \\
\hline \multirow{6}{*}{23} & $\mathrm{~L} \rightarrow \mathrm{T}$ & Uncharged Polar & Yes & 0.07 & Decrease & 8 & -1.52 \\
\hline & $\mathrm{L} \rightarrow \mathrm{S}$ & Uncharged Polar & No & 0.04 & Decrease & 9 & -1.55 \\
\hline & $\mathrm{L} \rightarrow \mathrm{H}$ & Basic & No & 0.03 & Decrease & 9 & -1.72 \\
\hline & $\mathrm{L} \rightarrow \mathrm{V}$ & Nonpolar & Yes & 0.43 & Decrease & 6 & -0.91 \\
\hline & $\mathrm{L} \rightarrow \mathrm{R}$ & Basic & Yes & 0.11 & Decrease & 5 & -1.15 \\
\hline & $\mathrm{L} \rightarrow \mathrm{Y}$ & Uncharged Polar & No & 0.02 & Decrease & 2 & -1.07 \\
\hline \multirow{5}{*}{24} & $\mathrm{~L} \rightarrow \mathrm{T}$ & Uncharged Polar & No & 0.04 & Decrease & 8 & -1.56 \\
\hline & $\mathrm{L} \rightarrow \mathrm{R}$ & Basic & No & 0.01 & Decrease & 5 & -1.19 \\
\hline & $\mathrm{L} \rightarrow \mathrm{M}$ & Nonpolar & Yes & 0.09 & Decrease & 6 & -0.97 \\
\hline & $\mathrm{L} \rightarrow \mathrm{I}$ & Nonpolar & Yes & 0.63 & Decrease & 7 & -1.10 \\
\hline & $\mathrm{L} \rightarrow \mathrm{E}$ & Acidic & No & 0.01 & Decrease & 6 & -1.41 \\
\hline
\end{tabular}


Table 1. Cont

\begin{tabular}{|c|c|c|c|c|c|c|c|}
\hline Position & Substitution & Characteristic & $\begin{array}{c}\text { SIFT } \\
\text { Tolerated }\end{array}$ & $\begin{array}{l}\text { SIFT } \\
\text { Score }\end{array}$ & $\begin{array}{l}\text { I-Mutant } \\
\text { Stability }\end{array}$ & RI & $\begin{array}{c}\text { DDG } \\
(\mathrm{kJ} / \mathrm{mol})\end{array}$ \\
\hline \multirow{5}{*}{25} & $G \rightarrow A$ & Nonpolar & Yes & 1.00 & Decrease & 1 & -0.57 \\
\hline & $G \rightarrow C$ & Nonpolar & Yes & 0.09 & Decrease & 6 & -0.98 \\
\hline & $\mathrm{G} \rightarrow \mathrm{T}$ & Uncharged Polar & Yes & 0.16 & Decrease & 7 & -0.79 \\
\hline & $G \rightarrow L$ & Nonpolar & Yes & 0.10 & Decrease & 6 & -0.53 \\
\hline & $G \rightarrow Y$ & Uncharged Polar & No & 0.03 & Decrease & 3 & -0.79 \\
\hline \multirow{4}{*}{26} & $\mathrm{I} \rightarrow \mathrm{S}$ & Uncharged Polar & Yes & 0.05 & Decrease & 9 & -1.33 \\
\hline & $\mathrm{I} \rightarrow \mathrm{L}$ & Nonpolar & Yes & 0.34 & Decrease & 8 & -0.63 \\
\hline & $\mathrm{I} \rightarrow \mathrm{D}$ & Acidic & No & 0.02 & Decrease & 8 & -1.29 \\
\hline & $\mathrm{I} \rightarrow \mathrm{N}$ & Uncharged Polar & No & 0.03 & Decrease & 8 & -1.26 \\
\hline \multirow{4}{*}{29} & $\mathrm{~L} \rightarrow \mathrm{D}$ & Acidic & No & 0.04 & Decrease & 8 & -1.82 \\
\hline & $\mathrm{L} \rightarrow \mathrm{F}$ & Nonpolar & Yes & 0.13 & Decrease & 8 & -1.20 \\
\hline & $\mathrm{L} \rightarrow \mathrm{K}$ & Basic & Yes & 0.08 & Decrease & 9 & -1.88 \\
\hline & $\mathrm{L} \rightarrow \mathrm{V}$ & Nonpolar & Yes & 0.28 & Decrease & 7 & -1.14 \\
\hline \multirow{5}{*}{30} & $\mathrm{~V} \rightarrow \mathrm{G}$ & Nonpolar & Yes & 0.55 & Decrease & 10 & -2.06 \\
\hline & $\mathrm{V} \rightarrow \mathrm{H}$ & Basic & No & 0.03 & Decrease & 10 & -1.87 \\
\hline & $\mathrm{V} \rightarrow \mathrm{E}$ & Acidic & Yes & 0.10 & Decrease & 8 & -1.54 \\
\hline & $\mathrm{V} \rightarrow \mathrm{Q}$ & Uncharged Polar & Yes & 0.07 & Decrease & 9 & -1.52 \\
\hline & $\mathrm{V} \rightarrow \mathrm{A}$ & Nonpolar & Yes & 1.00 & Decrease & 9 & -1.29 \\
\hline \multirow{4}{*}{33} & $\mathrm{I} \rightarrow \mathrm{T}$ & Uncharged Polar & No & 0.01 & Decrease & 9 & -1.46 \\
\hline & $\mathrm{I} \rightarrow \mathrm{F}$ & Nonpolar & Yes & 0.09 & Decrease & 9 & -1.00 \\
\hline & $\mathrm{I} \rightarrow \mathrm{K}$ & Basic & No & 0.00 & Decrease & 9 & -1.59 \\
\hline & $\mathrm{I} \rightarrow \mathrm{V}$ & Nonpolar & Yes & 1.00 & Decrease & 6 & -0.45 \\
\hline \multirow{4}{*}{34} & $\mathrm{I} \rightarrow \mathrm{T}$ & Uncharged Polar & No & 0.04 & Decrease & 9 & -1.50 \\
\hline & $I \rightarrow G$ & Uncharged Polar & Yes & 0.09 & Decrease & 9 & -2.09 \\
\hline & $\mathrm{I} \rightarrow \mathrm{F}$ & Nonpolar & Yes & 0.07 & Decrease & 9 & -0.98 \\
\hline & $\mathrm{I} \rightarrow \mathrm{L}$ & Nonpolar & Yes & 0.45 & Decrease & 8 & -0.83 \\
\hline \multirow{5}{*}{36} & $I \rightarrow G$ & Uncharged Polar & Yes & 0.17 & Decrease & 9 & -2.05 \\
\hline & $\mathrm{I} \rightarrow \mathrm{A}$ & Nonpolar & Yes & 0.38 & Decrease & 9 & -1.71 \\
\hline & $\mathrm{I} \rightarrow \mathrm{S}$ & Uncharged Polar & Yes & 0.11 & Decrease & 9 & -1.61 \\
\hline & $\mathrm{I} \rightarrow \mathrm{F}$ & Nonpolar & Yes & 0.14 & Decrease & 8 & -0.96 \\
\hline & $\mathrm{I} \rightarrow \mathrm{K}$ & Basic & Yes & 0.07 & Decrease & 9 & -1.64 \\
\hline \multirow{4}{*}{37} & $\mathrm{~L} \rightarrow \mathrm{T}$ & Uncharged Polar & No & 0.00 & Decrease & 9 & -1.86 \\
\hline & $\mathrm{L} \rightarrow \mathrm{M}$ & Nonpolar & No & 0.05 & Decrease & 8 & -1.13 \\
\hline & $\mathrm{L} \rightarrow \mathrm{I}$ & Nonpolar & No & 0.04 & Decrease & 9 & -1.30 \\
\hline & $\mathrm{L} \rightarrow \mathrm{V}$ & Nonpolar & Yes & 0.30 & Decrease & 8 & -1.13 \\
\hline \multirow{4}{*}{39} & $\mathrm{~V} \rightarrow \mathrm{A}$ & Nonpolar & Yes & 0.15 & Decrease & 9 & -1.24 \\
\hline & $\mathrm{V} \rightarrow \mathrm{D}$ & Acidic & No & 0.01 & Decrease & 9 & -1.57 \\
\hline & $\mathrm{V} \rightarrow \mathrm{K}$ & Basic & No & 0.03 & Decrease & 10 & -1.75 \\
\hline & $\mathrm{V} \rightarrow \mathrm{T}$ & Uncharged Polar & Yes & 0.12 & Decrease & 10 & -1.26 \\
\hline \multirow{5}{*}{40} & $\mathrm{P} \rightarrow \mathrm{T}$ & Uncharged Polar & No & 0.05 & Decrease & 8 & -0.99 \\
\hline & $\mathrm{P} \rightarrow \mathrm{A}$ & Nonpolar & Yes & 0.09 & Decrease & 8 & -1.10 \\
\hline & $\mathrm{P} \rightarrow \mathrm{D}$ & Acidic & No & 0.01 & Decrease & 8 & -1.23 \\
\hline & $\mathrm{P} \rightarrow \mathrm{N}$ & Uncharged Polar & No & 0.02 & Decrease & 8 & -1.38 \\
\hline & $\mathrm{P} \rightarrow \mathrm{F}$ & Nonpolar & Yes & 0.19 & Decrease & 8 & -0.80 \\
\hline \multirow{4}{*}{41} & $\mathrm{~L} \rightarrow \mathrm{Y}$ & Uncharged Polar & No & 0.00 & Decrease & 6 & -1.28 \\
\hline & $\mathrm{L} \rightarrow \mathrm{F}$ & Nonpolar & Yes & 0.06 & Decrease & 8 & -1.10 \\
\hline & $\mathrm{L} \rightarrow \mathrm{A}$ & Nonpolar & Yes & 0.06 & Decrease & 9 & -1.75 \\
\hline & $\mathrm{L} \rightarrow \mathrm{T}$ & Uncharged Polar & Yes & 0.09 & Decrease & 8 & -1.57 \\
\hline \multirow{3}{*}{44} & $\mathrm{~F} \rightarrow \mathrm{S}$ & Uncharged Polar & No & 0.03 & Decrease & 8 & -1.15 \\
\hline & $\mathrm{F} \rightarrow \mathrm{Y}$ & Uncharged Polar & Yes & 1.00 & Decrease & 2 & -0.74 \\
\hline & $\mathrm{F} \rightarrow \mathrm{I}$ & Nonpolar & Yes & 0.19 & Decrease & 5 & -0.80 \\
\hline \multirow{4}{*}{45} & $\mathrm{~T} \rightarrow \mathrm{I}$ & Nonpolar & Yes & 0.62 & Decrease & 6 & -0.77 \\
\hline & $\mathrm{T} \rightarrow \mathrm{N}$ & Uncharged Polar & No & 0.03 & Increase & 0 & -0.56 \\
\hline & $\mathrm{T} \rightarrow \mathrm{L}$ & Nonpolar & Yes & 0.28 & Decrease & 7 & -0.70 \\
\hline & $\mathrm{T} \rightarrow \mathrm{Y}$ & Uncharged Polar & No & 0.01 & Decrease & 3 & -0.51 \\
\hline
\end{tabular}


Table 2. Mutation Analysis of Vpu in I-Mutant 3.0 and SIFT.

\begin{tabular}{|c|c|c|c|c|c|c|c|}
\hline Position & Substitution & Characteristic & SIFT Tolerated & SIFT Score & I-Mutant Stability & RI & DDG (kJ/mol) \\
\hline \multirow{4}{*}{3} & $\mathrm{~S} \rightarrow \mathrm{T}$ & Uncharged Polar & Yes & 0.40 & Increase & 1 & -0.05 \\
\hline & $\mathrm{S} \rightarrow \mathrm{Y}$ & Uncharged Polar & Yes & 0.27 & Increase & 4 & -0.23 \\
\hline & $S \rightarrow D$ & Acidic & Yes & 0.49 & Increase & 6 & 0.06 \\
\hline & $\mathrm{S} \rightarrow \mathrm{M}$ & Basic & No & 0.04 & Increase & 4 & 0.00 \\
\hline \multirow{4}{*}{5} & $\mathrm{Q} \rightarrow \mathrm{V}$ & Nonpolar & Yes & 0.33 & Increase & 0 & 0.11 \\
\hline & $\mathrm{Q} \rightarrow \mathrm{E}$ & Acidic & Yes & 1.00 & Increase & 3 & -0.40 \\
\hline & $\mathrm{Q} \rightarrow \mathrm{L}$ & Nonpolar & Yes & 0.40 & Increase & 2 & 0.01 \\
\hline & $\mathrm{Q} \rightarrow \mathrm{G}$ & Nonpolar & Yes & 0.37 & Decrease & 7 & -0.80 \\
\hline \multirow{5}{*}{7} & $\mathrm{~L} \rightarrow \mathrm{S}$ & Uncharged Polar & Yes & 0.06 & Decrease & 9 & -1.38 \\
\hline & $\mathrm{L} \rightarrow \mathrm{Y}$ & Uncharged Polar & No & 0.01 & Decrease & 4 & -1.03 \\
\hline & $\mathrm{L} \rightarrow \mathrm{K}$ & Basic & No & 0.01 & Decrease & 8 & -1.30 \\
\hline & $\mathrm{L} \rightarrow \mathrm{V}$ & Nonpolar & Yes & 0.23 & Decrease & 6 & -0.72 \\
\hline & $\mathrm{L} \rightarrow \mathrm{I}$ & Nonpolar & Yes & 0.52 & Decrease & 8 & -0.94 \\
\hline \multirow{4}{*}{8} & $\mathrm{~A} \rightarrow \mathrm{T}$ & Uncharged Polar & No & 0.04 & Decrease & 9 & -1.22 \\
\hline & $A \rightarrow G$ & Nonpolar & Yes & 0.93 & Decrease & 9 & -1.62 \\
\hline & $\mathrm{A} \rightarrow \mathrm{S}$ & Uncharged Polar & Yes & 0.20 & Decrease & 10 & -1.32 \\
\hline & $\mathrm{A} \rightarrow \mathrm{N}$ & Uncharged Polar & No & 0.02 & Decrease & 9 & -1.21 \\
\hline \multirow{5}{*}{11} & $\mathrm{~A} \rightarrow \mathrm{F}$ & Nonpolar & No & 0.00 & Decrease & 9 & -0.77 \\
\hline & $\mathrm{A} \rightarrow \mathrm{S}$ & Uncharged Polar & Yes & 0.09 & Decrease & 10 & -1.18 \\
\hline & $A \rightarrow G$ & Nonpolar & Yes & 0.14 & Decrease & 10 & -1.51 \\
\hline & $\mathrm{A} \rightarrow \mathrm{E}$ & Acidic & Yes & 0.06 & Decrease & 9 & -1.16 \\
\hline & $\mathrm{A} \rightarrow \mathrm{T}$ & Uncharged Polar & No & 0.01 & Decrease & 9 & -1.09 \\
\hline \multirow{4}{*}{14} & $\mathrm{~V} \rightarrow \mathrm{A}$ & Nonpolar & Yes & 0.10 & Decrease & 4 & -0.82 \\
\hline & $\mathrm{V} \rightarrow \mathrm{L}$ & Nonpolar & No & 0.02 & Decrease & 6 & -0.96 \\
\hline & $\mathrm{V} \rightarrow \mathrm{T}$ & Uncharged Polar & No & 0.01 & Decrease & 10 & -1.08 \\
\hline & $\mathrm{V} \rightarrow \mathrm{I}$ & Nonpolar & Yes & 0.14 & Decrease & 8 & -0.69 \\
\hline \multirow{6}{*}{15} & $\mathrm{~A} \rightarrow \mathrm{T}$ & Uncharged Polar & Yes & 0.16 & Decrease & 9 & -1.20 \\
\hline & $\mathrm{A} \rightarrow \mathrm{F}$ & Nonpolar & No & 0.00 & Decrease & 9 & -0.77 \\
\hline & $A \rightarrow G$ & Nonpolar & No & 0.02 & Decrease & 10 & -1.59 \\
\hline & $\mathrm{A} \rightarrow \mathrm{S}$ & Uncharged Polar & No & 0.03 & Decrease & 10 & -1.28 \\
\hline & $\mathrm{A} \rightarrow \mathrm{E}$ & Acidic & Yes & 0.13 & Decrease & 9 & -1.26 \\
\hline & $\mathrm{A} \rightarrow \mathrm{N}$ & Uncharged Polar & No & 0.01 & Decrease & 9 & -1.31 \\
\hline \multirow{4}{*}{17} & $\mathrm{I} \rightarrow \mathrm{A}$ & Nonpolar & No & 0.01 & Decrease & 4 & -1.12 \\
\hline & $\mathrm{I} \rightarrow \mathrm{L}$ & Nonpolar & Yes & 0.17 & Decrease & 1 & -0.34 \\
\hline & $\mathrm{I} \rightarrow \mathrm{T}$ & Uncharged Polar & No & 0.01 & Decrease & 8 & -1.12 \\
\hline & $\mathrm{I} \rightarrow \mathrm{S}$ & Uncharged Polar & Yes & 0.09 & Decrease & 8 & -1.31 \\
\hline \multirow{5}{*}{19} & $\mathrm{~A} \rightarrow \mathrm{T}$ & Uncharged Polar & Yes & 0.09 & Decrease & 9 & -1.09 \\
\hline & $\mathrm{A} \rightarrow \mathrm{N}$ & Uncharged Polar & No & 0.00 & Decrease & 9 & -1.20 \\
\hline & $A \rightarrow G$ & Nonpolar & No & 0.01 & Decrease & 9 & -1.56 \\
\hline & $\mathrm{A} \rightarrow \mathrm{V}$ & Nonpolar & Yes & 0.15 & Decrease & 7 & -0.54 \\
\hline & $A \rightarrow Q$ & Uncharged Polar & No & 0.01 & Decrease & 9 & -1.10 \\
\hline \multirow{4}{*}{23} & $\mathrm{~W} \rightarrow \mathrm{K}$ & Basic & No & 0.00 & Decrease & 9 & -1.11 \\
\hline & $\mathrm{W} \rightarrow \mathrm{Y}$ & Uncharged Polar & No & 0.00 & Decrease & 7 & -0.86 \\
\hline & $\mathrm{W} \rightarrow \mathrm{R}$ & Basic & No & 0.00 & Decrease & 7 & -0.69 \\
\hline & $\mathrm{W} \rightarrow \mathrm{Q}$ & Uncharged Polar & No & 0.00 & Decrease & 9 & -1.07 \\
\hline \multirow{4}{*}{25} & $\mathrm{I} \rightarrow \mathrm{V}$ & Nonpolar & No & 0.04 & Decrease & 7 & -0.32 \\
\hline & $\mathrm{I} \rightarrow \mathrm{M}$ & Basic & No & 0.01 & Decrease & 8 & -0.81 \\
\hline & $\mathrm{I} \rightarrow \mathrm{K}$ & Basic & Yes & 0.05 & Decrease & 9 & -1.52 \\
\hline & $\mathrm{I} \rightarrow \mathrm{L}$ & Nonpolar & Yes & 0.24 & Decrease & 5 & -0.39 \\
\hline \multirow{4}{*}{27} & $\mathrm{~F} \rightarrow \mathrm{S}$ & Uncharged Polar & No & 0.03 & Decrease & 8 & -1.15 \\
\hline & $\mathrm{F} \rightarrow \mathrm{Y}$ & Uncharged Polar & Yes & 1.00 & Decrease & 2 & -0.74 \\
\hline & $\mathrm{F} \rightarrow \mathrm{I}$ & Nonpolar & Yes & 0.19 & Decrease & 5 & -0.80 \\
\hline & $\mathrm{F} \rightarrow \mathrm{G}$ & Nonpolar & Yes & 0.08 & Decrease & 7 & -1.35 \\
\hline \multirow{4}{*}{31} & $\mathrm{R} \rightarrow \mathrm{T}$ & Uncharged Polar & No & 0.01 & Decrease & 8 & -0.53 \\
\hline & $\mathrm{R} \rightarrow \mathrm{A}$ & Nonpolar & No & 0.01 & Decrease & 7 & -0.49 \\
\hline & $\mathrm{R} \rightarrow \mathrm{K}$ & Basic & Yes & 0.54 & Decrease & 9 & -0.75 \\
\hline & $\mathrm{R} \rightarrow \mathrm{L}$ & Nonpolar & No & 0.01 & Decrease & 8 & -0.42 \\
\hline \multirow{4}{*}{32} & $\mathrm{~K} \rightarrow \mathrm{E}$ & Acidic & Yes & 0.15 & Increase & 2 & -0.22 \\
\hline & $\mathrm{K} \rightarrow \mathrm{I}$ & Nonpolar & No & 0.00 & Increase & 1 & -0.23 \\
\hline & $\mathrm{K} \rightarrow \mathrm{R}$ & Basic & Yes & 0.15 & Increase & 5 & 0.03 \\
\hline & $\mathrm{K} \rightarrow \mathrm{A}$ & Nonpolar & No & 0.01 & Increase & 6 & -0.06 \\
\hline
\end{tabular}




\subsection{Aggregation Potential Prediction of Tetherin}

There are several online tools and web servers available to estimate amyloidogenic regions from protein sequences based on complex biological mechanisms linked to amyloidosis and are based on consider diverse physicochemical features, such as charge, secondary-structure propensity, and hydrophobicity [41,42]. Formation of cross $\beta$-sheet arrangement is a principle characteristic of an amyloid and has a prime contribution in identification of potentially aggregating regions [43]. The amyloidogenic region prediction of tetherin was done in Fold Amyloid [44], AGGRESCAN [45], TANGO [46], WALTZ [47], MetAmyl [48], AMYLPRED2 [49], and PASTA [50] programs that differ in their algorithms, and each has own applications for predictions of aggregation-prone sites or specific segments of proteins that can tend to aggregate. These tools integrate diverse properties of proteins accountable for amyloidogenicity, such as hydrophobicity, aggregation propensity scale, $\beta$-strand contiguity, average packing density, hexapeptide conformational energy, and possible conformational switches between $\alpha$-helix and $\beta$-sheet [51-53]. The secondary structure prediction for tetherin is performed in the Chou-Fasman server (http://cho-fas.sourceforge.net/) to derive the relative frequencies of each amino acid in $\alpha$-helices, $\beta$-sheets, and coils based on known protein crystal structures solved with X-ray crystallography [54]. The TANGO server predicts aggregation nucleating regions in proteins, as well as the effect of mutations and environmental conditions on the aggregation propensity of these regions [46]. Identification of discordant region in tetherin was performed by estimating the amino acid propensity values from the TANGO (http://tango.crg.es/n) web server. It provides propensities for each amino acid in the sequence to form either $\alpha$-helix or $\beta$-sheet. The possible conformational switches in the query sequence were predicted based on secondary structure. Conformational switches are regions that can alter their shape in response to certain input signals either ligand binding, chemical binding, or environmental conditions [55]. The amino acids in such stretches have propensities to form both helix and sheet encompassing a $\alpha$-helix/ $\beta$-sheet discordant region.

\section{Results}

\subsection{Modeled Protein Structures and Evaluation}

The 3D structures of blood- and brain-derived Vpu and consensus Vpu were modeled using the SWISS-MODEL server and assessed the overall quality in PROSA giving a z-score of 0.52 for Vpu from blood isolate, -0.14 for Vpu from brain isolate, and 0.52 for the consensus Vpu structure. The z-score for all the modeled Vpu structures were found to fall in the range of scores typically found for native proteins of similar size. The Ramachandran plot statistics of the modeled Vpu structures presented in Table 3 had over $90 \%$ of residues in the most favorable region, indicating good overall model quality. The minimized energy as calculated in Swiss PDB Viewer (SPDBV) was about -592.97 $\mathrm{kcal} / \mathrm{mol}$ for blood-derived $\mathrm{Vpu}$; for brain-derived $\mathrm{Vpu}$, it was $-615.91 \mathrm{kcal} / \mathrm{mol}$, and, for the consensus Vpu structure, it was $-763.86 \mathrm{kcal} / \mathrm{mol}$.

Table 3. Ramachandran plot statistics of blood- and brain-derived modeled Vpu structures and modeled consensus Vpu structure presenting overall model quality.

\begin{tabular}{cccccc}
\hline $\begin{array}{c}\text { Protein } \\
\text { Modeled }\end{array}$ & $\begin{array}{c}\text { Residues in Most } \\
\text { Favored Region }\end{array}$ & $\begin{array}{c}\text { Residues in } \\
\text { Additional } \\
\text { Allowed Region }\end{array}$ & $\begin{array}{c}\text { Residues in } \\
\text { Generously } \\
\text { Allowed Region }\end{array}$ & $\begin{array}{c}\text { Residues in } \\
\text { Disallowed } \\
\text { Region }\end{array}$ & $\begin{array}{c}\text { No. of } \\
\text { Glycines }\end{array}$ \\
\hline $\begin{array}{c}\text { Blood-derived } \\
\text { HIV-1 Vpu }\end{array}$ & $94.7 \%$ & $4.0 \%$ & $1.3 \%$ & $0.0 \%$ & 3 \\
\hline $\begin{array}{c}\text { Brain-derived } \\
\text { HIV-1 Vpu }\end{array}$ & $90.5 \%$ & $8.1 \%$ & $1.4 \%$ & $0.0 \%$ & 4 \\
\hline $\begin{array}{c}\text { Consensus } \\
\text { HIV-Vpu }\end{array}$ & $92.0 \%$ & $6.7 \%$ & $1.3 \%$ & $0.0 \%$ & 3 \\
\hline
\end{tabular}




\subsection{Docking Studies of Wild-Type Structures}

Protein-protein interactions performed for the modeled Vpu structures with tetherin in Hex provided around 30-50 possible docked confirmations that were then manually visualized for their anti-parallel orientation and interacting positions and were tested in the PIC server for estimating the interacting residues. Our focus had been mainly on deriving the maximum interacting residues between the TM regions of both proteins Vpu and tetherin, as it has been reported that the antagonism of Vpu to the anti-viral activity of tetherin is by intermolecular interactions between the helix-helix TM domains of both the proteins $[12,14]$. Thus, such complexes were manually selected and submitted to PRODIGY for evaluating the finest docked confirmation with maximum interacting residues and best binding affinity. The value of the dissociation constant $(\mathrm{Kd})$ is calculated at $37^{\circ} \mathrm{C}$ in PRODIGY. The selected complexes, their binding affinities, and $\mathrm{Kd}$ values are represented in Table 4 .

Table 4. Presentation of binding affinity and dissociation constant $(\mathrm{Kd})$ calculated from PROtein binDIng enerGY prediction (PRODIGY) and potential energy computed in SPDBV for Vpu-tetherin complexes.

\begin{tabular}{|c|c|c|c|c|}
\hline Protein-Protein Complex & Model Selected & $\begin{array}{l}\text { Binding Affinity } \\
\Delta \mathrm{G} \text { (kcal/mol) }\end{array}$ & $\begin{array}{c}\text { Dissociation } \\
\text { Constant } \mathrm{Kd}(\mathrm{M}) \\
\text { at } 37.0^{\circ} \mathrm{C}\end{array}$ & $\begin{array}{c}\text { Potential Energy } \\
\text { in SPDBV } \\
(\mathrm{kcal} / \mathrm{mol})\end{array}$ \\
\hline $\begin{array}{l}\text { Wild-type blood-derived } \\
\text { Vpu-tetherin }\end{array}$ & $\begin{array}{c}\text { Model } \\
\text { dock0009.pdb }\end{array}$ & -5.0 & $6.4 \times 10^{-4}$ & -314.17 \\
\hline $\begin{array}{c}\text { Wild-type brain derived } \\
\text { Vpu-tetherin }\end{array}$ & $\begin{array}{c}\text { Model } \\
\text { dock0001.pdb }\end{array}$ & -3.8 & $2.0 \times 10^{-3}$ & -148.65 \\
\hline $\begin{array}{l}\text { Wild-type consensus } \\
\text { Vpu-tetherin }\end{array}$ & $\begin{array}{c}\text { Model } \\
\text { dock0007.pdb }\end{array}$ & -4.3 & $9.8 \times 10^{-4}$ & -261.08 \\
\hline
\end{tabular}

The residue pairs forming hydrophobic interactions within $5 \AA$ in wild-type blood-derived Vpu-tetherin complex having a binding affinity $(\Delta \mathrm{G})$ of $-5.0 \mathrm{kcal} / \mathrm{mol}$ and $\mathrm{Kd}$ of $6.4 \times 10^{-4} \mathrm{M}$ were obtained for A8-I42, A8-V39, I9-I42, I9-L41, I9-P40, I9-V39, V10-I43, V10-I42, V10-P40, V10-V39, A11-V39, L12-V39, L12-I36, L12-V35, V13-P40, V13-V39, V13-L37, V13-I36, V14-I36, A15-I36, I17-I36, A19-L32, I20-I33, I20-L32, I20-L29, W23-L29, W23-I28, I25-L22, F27-I28, F27-I26, F27-L24, F27-L23, F27-L22, I28-I26, and I28-L22, as presented in Figure 2. The anti-parallel interactions between the TM domains of blood derived Vpu and tetherin is are presented in Figure 3. The complex had a potential energy of $-314.17 \mathrm{kcal} / \mathrm{mol}$ as computed by force field parameters. The residue pairs forming hydrophobic interactions within $5 \AA$ in wild-type brain-derived Vpu-tetherin complex having a binding affinity $(\Delta \mathrm{G})$ of $-3.8 \mathrm{kcal} / \mathrm{mol}$ and $\mathrm{Kd}$ of $2.0 \times 10^{-3} \mathrm{M}$ were obtained for V10-P40, V10-V39, V10-L37, V10-I36, A11-L37, V13-I36, V14-L37, V14-I36, V14-I34, V14-I33, I17-I36, I17-I33, I17-L32, I17-L29, I18-I33, I18-V30, I18-L29, I20-L29, V21-V30, V21-L29, V21-I28, V21-I26, I25-L23, I25-L22, and I39-L22, as presented in Figure 2. The anti-parallel interactions between the TM domains of brain derived $\mathrm{Vpu}$ and tetherin are presented in Figure 4. A potential energy computed by force field parameters was $-148.65 \mathrm{kcal} / \mathrm{mol}$ for this complex. The residue pairs forming hydrophobic interactions within $5 \AA$ in wild-type consensus Vpu-tetherin complex having a binding affinity $(\Delta \mathrm{G})$ of -4.3 $\mathrm{kcal} / \mathrm{mol}$ and $\mathrm{Kd}$ of $9.8 \times 10^{-4} \mathrm{M}$ were obtained for L7-F44, L7-I43, L7-L41, A8-I43, A8-P40, V10-P40, V10-L37, A11-L37, L12-L37, V14-L37, V14-I36, V14-I34, V14-I33, A15-L37, I17-I33, I17-L29, I18-I34, I18-I33, I18-V30, V21-V30, V21-L29, V21-I26, V22-V30, I25-I26, I25-L24, I25-L23, V26-L23, and I28-L23, as presented in Figure 2. The complex had a potential energy of $-261.08 \mathrm{kcal} / \mathrm{mol}$, as computed by force field parameters. The anti-parallel interaction between the TM domains of consensus Vpu and tetherin are presented in Figure 5. There were no direct bonds observed in the docked complexes. 


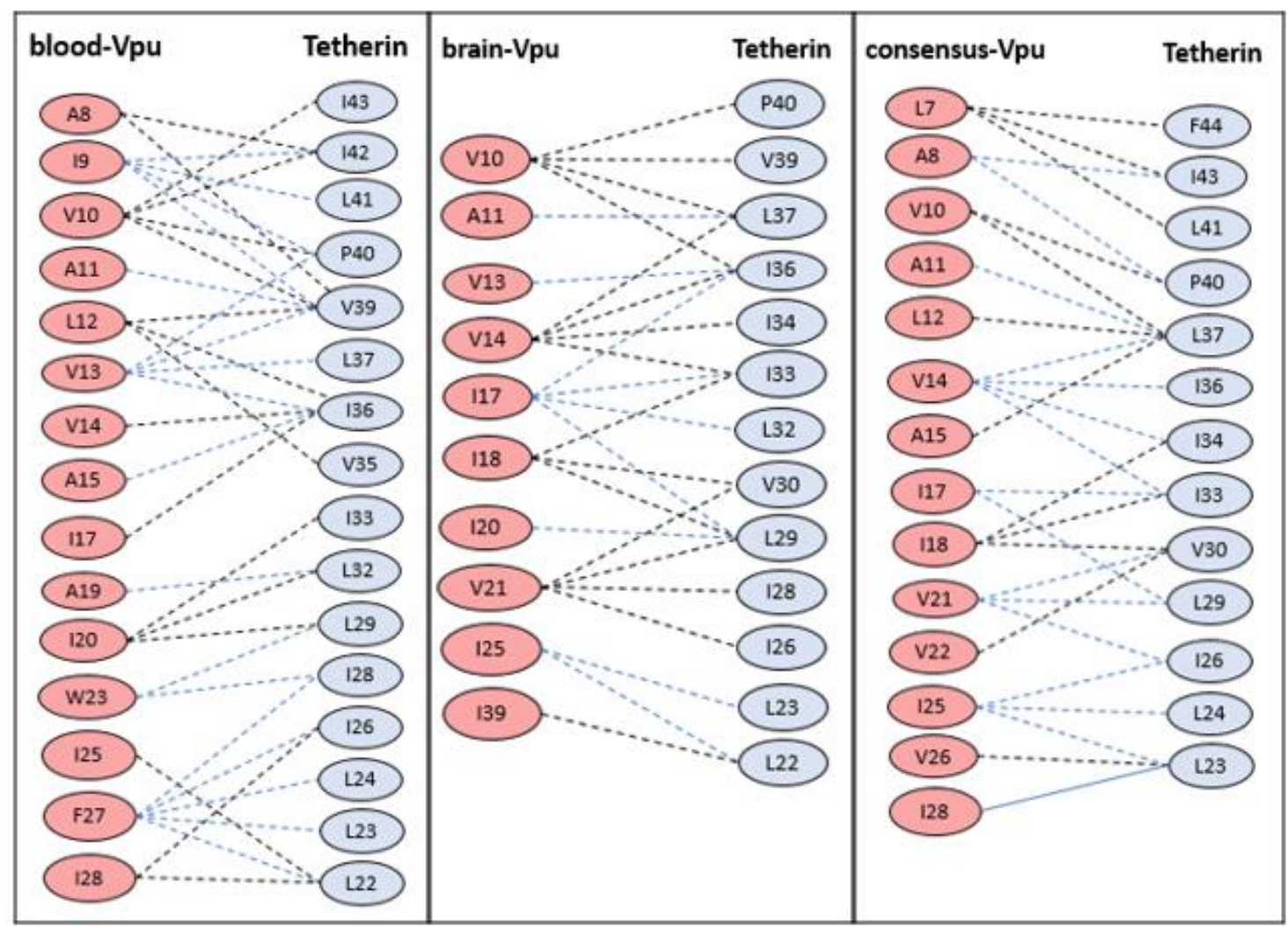

Figure 2. The hydrophobic interactions within $5 \AA$ for wild-type blood-derived Vpu-tetherin complex, wild-type brain-derived Vpu-tetherin complex, and wild-type consensus Vpu-tetherin complex.
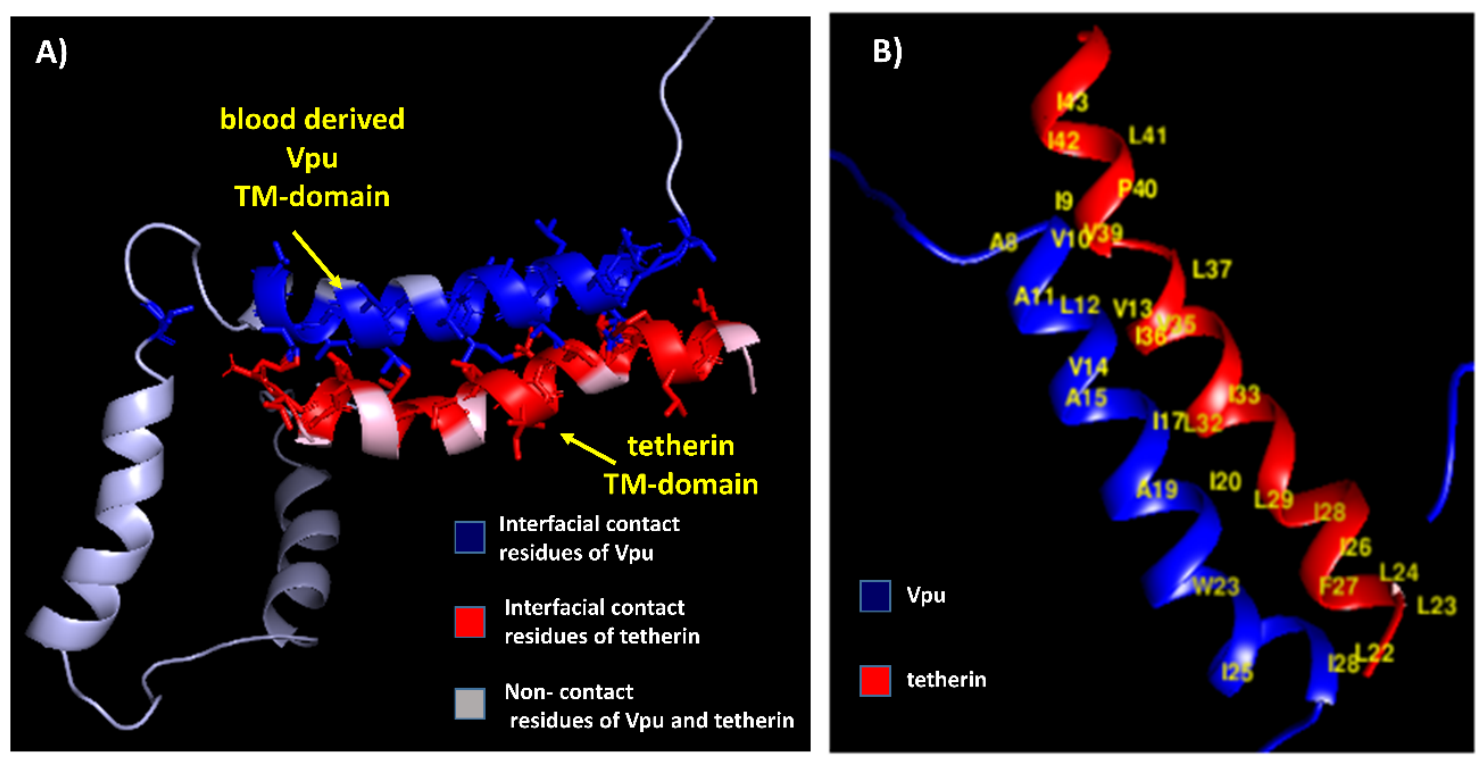

Figure 3. (A) Blood-derived Vpu-tetherin complex with interfacial contact residues of $\mathrm{Vpu}$ in blue, interfacial residues of tetherin in red, and non-contact residues in grey. (B) Residues forming hydrophobic interactions (labeled in yellow) between blood-derived Vpu and tetherin. 

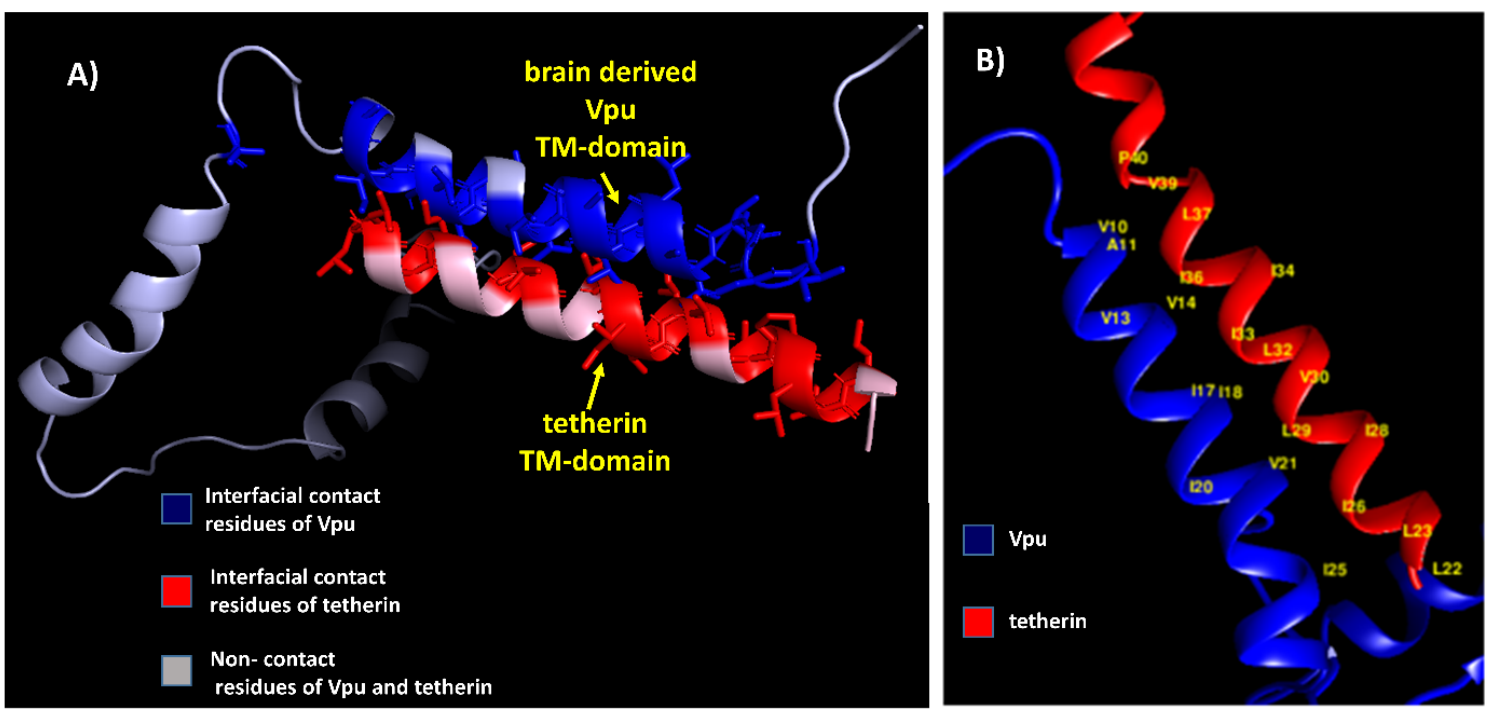

Figure 4. (A) Brain-derived Vpu-tetherin complex with interfacial contact residues of Vpu in blue, interfacial residues of tetherin in red, and non-contact residues in grey. (B) Residues forming hydrophobic interactions (labeled in yellow) between brain-derived Vpu and tetherin.
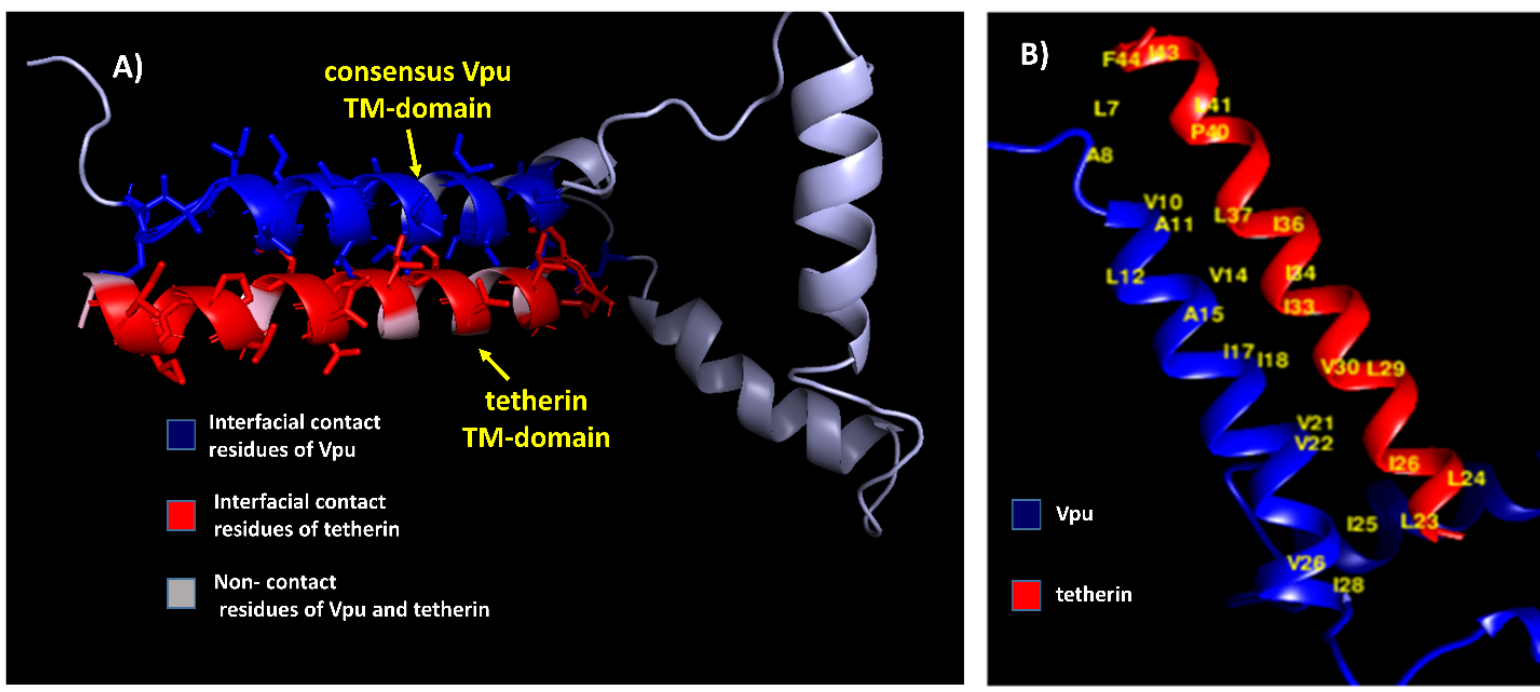

Figure 5. (A) Consensus Vpu-tetherin complex with interfacial contact residues of Vpu in blue, interfacial residues of tetherin red, and non-contact residues in grey. (B) Residues forming hydrophobic interactions (labeled in yellow) between consensus Vpu and tetherin.

\subsection{Docking Studies of Mutant Structures}

The amino acids of tetherin at positions L22, L23, L24, G25, I26, L29, V30, I33, I34, I36, L37, V39, P40, L41, F44, and T45 and those of blood-derived Vpu at positions S3, Q5, L7, A8, A11, V14, A15, I17, A19, W23, F27, R31, and K32 were subjected to synonymous and non-synonymous mutations, and their results for stability and tolerance is given in Tables 1 and 2. Based on these scores, few mutations were shortlisted, and tetherin and Vpu structures were mutated in Chimera. The protein-protein interactions were performed and analyzed for these mutant structures. The selected mutations of $\mathrm{Vpu}$ and tetherin, interacting residues between them, and binding energy of the complexes are listed in Tables 5 and 6. All the mutated structures of tetherin and Vpu exhibited a decrease in binding energies in comparison with the wild-type blood-derived Vpu-tetherin complex. The single and triple mutations that resulted in reduced binding affinities and fewer hydrophobic connections as compared to other mutations are represented in Figures 6 and 7. It was observed that the following set of residues, A11, V13, V14, A15, I17, I25, and I28, of Vpu and L22, L23, L24, I33, V30, I36, L37, P40, I43, and F44 of 
tetherin were found interacting in most of the mutant structures, indicating their critical role in the formation of binding complexes that remain unaffected despite of mutations.

Table 5. This table represents the positions of mutations performed in tetherin, the best tetherin-Vpu interaction model (docked model) in Hex, binding affinity of the respective docked complexes, and their $\mathrm{Kd}$ values.

\begin{tabular}{|c|c|c|c|c|c|}
\hline Name & Type of Mutation & $\begin{array}{l}\text { Best Model } \\
\text { Selected }\end{array}$ & $\begin{array}{c}\text { Binding Affinity } \\
\Delta \mathrm{G} \text { (kcal/mol) }\end{array}$ & $\mathrm{Kd}(\mathrm{M})$ at $37.0^{\circ} \mathrm{C}$ & $\begin{array}{c}\text { Energy (iMutant) } \\
\text { (kcal/mol) }\end{array}$ \\
\hline Tetherin & Wild-type & 09 & -5.0 & $6.4 \times 10^{-4}$ & NA \\
\hline M1 & L22S & 10 & -3.6 & $2.9 \times 10^{-3}$ & Decrease by -1.31 \\
\hline M2 & L22Y & 06 & -3.9 & $3.5 \times 10^{-1}$ & Decrease by -2.06 \\
\hline M3 & L23T & 05 & -2.9 & $3.4 \times 10^{-2}$ & Decrease by -1.52 \\
\hline M4 & L23Y & 01 & -2.6 & $1.2 \times 10^{-3}$ & Decrease by -2.06 \\
\hline M5 & L24F & 02 & -3.0 & $6.0 \times 10^{-4}$ & Decrease by -1.00 \\
\hline M6 & L24M & 08 & -3.2 & $3.2 \times 10^{-3}$ & Decrease by -1.52 \\
\hline M7 & $\mathrm{L} 24 \mathrm{~T}$ & 04 & -2.2 & $2.9 \times 10^{-3}$ & Decrease by -2.86 \\
\hline M8 & G25A & 10 & -3.6 & $4.1 \times 10^{-3}$ & Decrease by -0.57 \\
\hline M9 & I26S & 08 & -3.5 & $3.9 \times 10^{-5}$ & Decrease by -1.33 \\
\hline M10 & L29Q & 06 & -3.9 & $4.4 \times 10^{-3}$ & Decrease by -0.87 \\
\hline M11 & V30G & 07 & -4.1 & $3.9 \times 10^{-2}$ & Decrease by -2.06 \\
\hline M12 & I33T & 07 & -3.5 & $4.2 \times 10^{-3}$ & Decrease by -1.46 \\
\hline M13 & I34T & 08 & -4.3 & $4.5 \times 10^{-5}$ & Decrease by -1.50 \\
\hline M14 & $\mathrm{I} 36 \mathrm{G}$ & 08 & -4.1 & $3.5 \times 10^{-1}$ & Decrease by -2.05 \\
\hline M15 & L37T & 17 & -4.3 & $3.8 \times 10^{-2}$ & Decrease by -1.86 \\
\hline M16 & V39 & 14 & -3.6 & $4.5 \times 10^{-1}$ & Decrease by -2.25 \\
\hline M17 & P40T & 11 & -2.7 & $1.9 \times 10^{-3}$ & Decrease by -0.99 \\
\hline M18 & L41Y & 17 & -3.0 & $3.9 \times 10^{-6}$ & Decrease by -1.28 \\
\hline M19 & T45I & 06 & -2.5 & $2.7 \times 10^{-3}$ & Decrease by -0.77 \\
\hline M20 & L22S, F44Y, L37I & 01 & -2.2 & $1.8 \times 10^{-2}$ & NA \\
\hline M21 & L23T, L37T, T45I & 05 & -2.9 & $2.3 \times 10^{-3}$ & NA \\
\hline
\end{tabular}

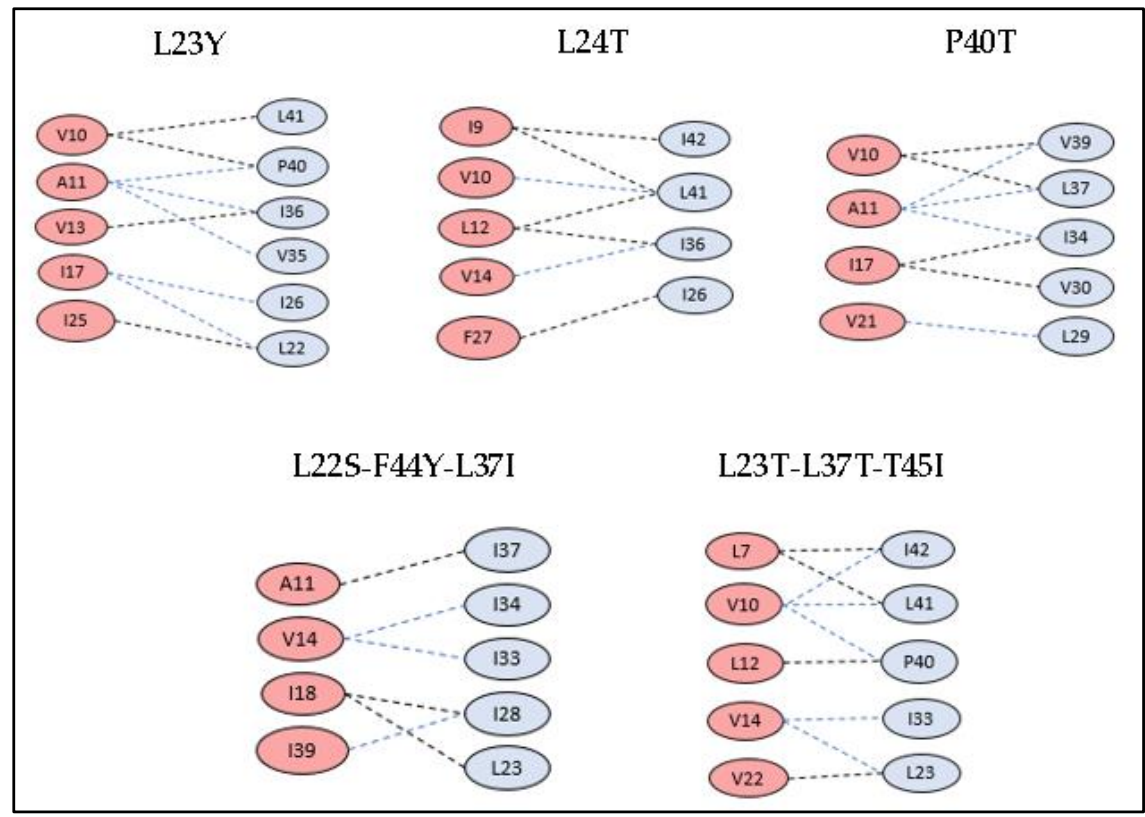

Figure 6. The hydrophobic interactions within $5 \AA$ for tetherin mutants having reduced binding affinities and minimal hydrophobic connections. 
Table 6. This table represents the positions of mutations performed in $\mathrm{Vpu}$, the best tetherin-Vpu interaction model (docked model) in Hex, binding affinity of the respective docked complexes, and their $\mathrm{Kd}$ values.

\begin{tabular}{cccccc}
\hline Name & Type of Mutation & $\begin{array}{c}\text { Best Model } \\
\text { Selected }\end{array}$ & $\begin{array}{c}\text { Binding Affinity } \\
\Delta \mathbf{G} \text { (kcal/mol) }\end{array}$ & Kd (M) at 37.0 ${ }^{\circ} \mathbf{C}$ & $\begin{array}{c}\text { Energy (iMutant) } \\
\text { (kcal/mol) }\end{array}$ \\
\hline Vpu_blood & Wild-type & 09 & -5.0 & $6.4 \times 10^{-4}$ & NA \\
\hline M1 & S03Y & 12 & -3.6 & $3.3 \times 10^{-3}$ & Increase by -0.23 \\
\hline M2 & Q05V & 19 & -2.9 & $3.4 \times 10^{-4}$ & Increase by -0.11 \\
\hline M3 & L07S & 17 & -3.5 & $3.8 \times 10^{-2}$ & Decrease by -1.38 \\
\hline M4 & A08T & 07 & -4.9 & $4.4 \times 10^{-4}$ & Decrease by -1.22 \\
\hline M5 & A11F & 12 & -3.2 & $4.1 \times 10^{-5}$ & Decrease by -0.77 \\
\hline M6 & A11L & 08 & -3.6 & $3.2 \times 10^{-5}$ & Decrease by -0.78 \\
\hline M7 & V14K & 04 & -2.9 & $3.4 \times 10^{-4}$ & Decrease by -1.28 \\
\hline M8 & A15L & 15 & -3.5 & $3.9 \times 10^{-1}$ & Decrease by -1.20 \\
\hline M9 & A15T & 07 & -3.6 & $3.7 \times 10^{-1}$ & Decrease by -0.94 \\
\hline M10 & I17A & 08 & -4.5 & $3.5 \times 10^{-4}$ & Decrease by -1.12 \\
\hline M11 & A19T & 16 & -3.5 & $4.4 \times 10^{-2}$ & Decrease by -1.09 \\
\hline M12 & A19H & 14 & -2.5 & $2.9 \times 10^{-3}$ & Decrease by -1.36 \\
\hline M13 & A19F & 06 & -4.7 & $4.1 \times 10^{-2}$ & Decrease by -0.86 \\
\hline M14 & A19L & 06 & -4.7 & $4.9 \times 10^{-2}$ & Decrease by -0.92 \\
\hline M15 & W23K & 06 & -4.1 & $5.1 \times 10^{-4}$ & Decrease by -1.11 \\
\hline M16 & W23L & 05 & -3.4 & $3.7 \times 10^{-1}$ & Decrease by -0.56 \\
\hline M17 & W23Y & 07 & -2.1 & $2.1 \times 10^{-3}$ & Decrease by -0.97 \\
\hline M18 & R31A & 17 & -3.3 & $4.8 \times 10^{-4}$ & Decrease by -0.49 \\
\hline M19 & K32A & 04 & -4.2 & $4.9 \times 10^{-3}$ & Increase by -0.06 \\
\hline M20 & V10K A11L, A19T & 01 & -2.6 & $2.1 \times 10^{-5}$ & NA \\
\hline M21 & V14T, I18T, I26S & 07 & -2.7 & $2.2 \times 10^{-4}$ & NA \\
\hline M22 & A11T, V14L, A15T & 02 & -2.3 & $2.8 \times 10^{-2}$ & NA \\
\hline
\end{tabular}

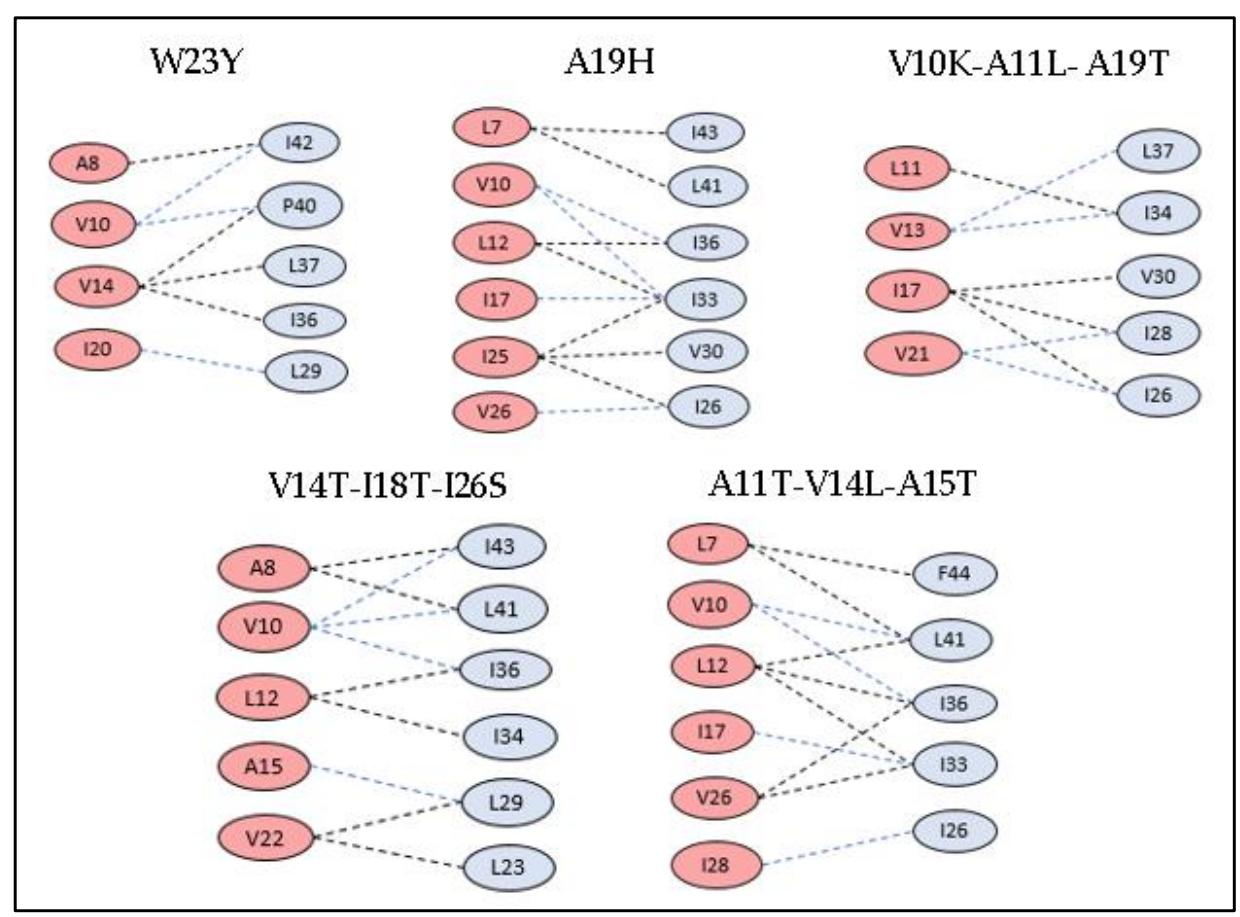

Figure 7. The hydrophobic interactions within $5 \AA$ for Vpu mutants having reduced binding affinities and minimal hydrophobic connections. 


\subsection{Amyloidogenicity Prediction of Tetherin}

The aggregation prediction results of tetherin showed the presence of amyloid forming regions at positions ranging from 21 to 47 , as well as from 161 to 176 derived out of consensus output from at least six out of seven servers (Table 7). The region 21-47 forms the TM region that is known to interact with $\mathrm{Vpu}$ [56]. The various amyloid prediction servers employ different algorithms based on aggregation propensity scale, expected packing density, physicochemical properties of secondary structure, $\beta$ strand contiguity, and formation of possible conformational switches and presents the potential aggregating regions as hotspots, as represented in Table 7. Parallel aggregation within the predicted region of 21-47 is observed in PASTA 2.0 with energy of -20.54 PASTA Energy Units (1 PEU $=1.192 \mathrm{kcal} / \mathrm{mol}$ ), as shown in Figure 8A. The program predicts region in query sequence likely to form $\beta$-strand inter-molecular pairing, thus identifying the aggregation probability of the region [50]. The aggregation energy indicates a low energy cross $\beta$-structure conformation of predicted stretch signifying a stabilized assembly, while the other predicted stretch of 161-176 comprises a disordered region, as indicated in Figure 8B, presenting aggregation and disorder profile of tetherin. Discordant regions predicted by secondary structure in tetherein lies at positions 28-34, 42-46, 68-69, 93-96, 144-148, and 167-175. The propensity values for tetherin as predicted in Chou-Fasman for identified aggregating region $21-47$ were $96.3 \%$ for helix and $88.9 \%$ for sheet and for region $161-176$ were $75 \%$ for helix and $37.5 \%$ for sheet (Table 8). Region $21-47$ of tetherin does show the presence of amino acids with high propensity for both helix and sheet. The aggregating regions in tetherin are predicted at positions 22-38 and 168-179. The helix and sheet aggregation plots are presented in Figure 9.

A) Energy -20.548414 pairing segments

Probability $(\mathrm{k}, \mathrm{m})$

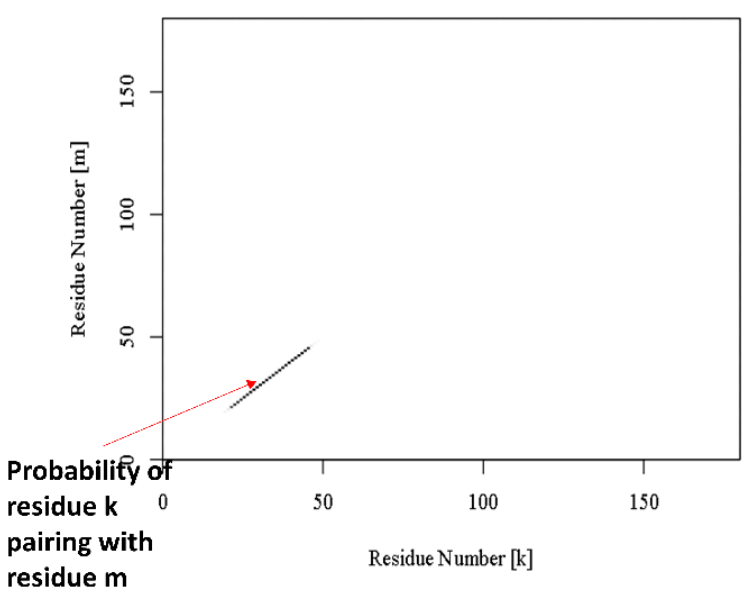

B) Aggregation and Disorder Profile

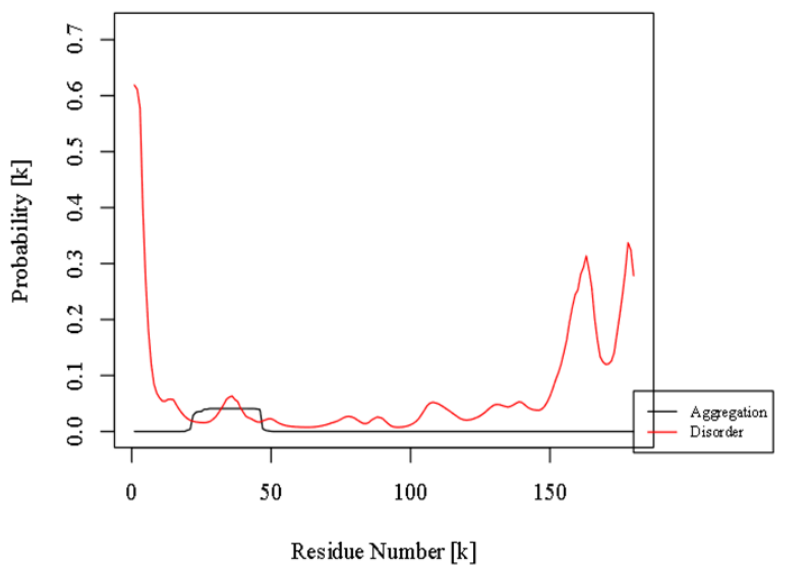

Figure 8. (A) Pairing results in PASTA2.0 for tetherin. (B) Aggregation and disorder profile of tetherin-derived in PASTA2.0. 
Table 7. Aggregating regions predicted in tetherin by seven servers. Consensus results are highlighted as regions predicted by at least six of the seven servers.

\begin{tabular}{|c|c|c|c|c|c|c|c|c|c|}
\hline $\begin{array}{l}\text { Protein Name } \\
\text { Accession }\end{array}$ & Position & Consensus Predicted Amyloid Regions & Fold Amyloid & Aggrescan $^{* *} \pm$ & Tango ${ }^{* * \pm}$ & MetAmyl & $\begin{array}{c}\text { AMYLPRED2 } \\
\text { Consensus }\end{array}$ & Waltz ** & PASTA \\
\hline TetherinQ10589 & $\begin{array}{c}21-47 \\
161-176\end{array}$ & $\begin{array}{l}\text { >sp|Q10589|BST2_HUMAN Bone marrow stromal antigen } 2 \\
\text { OS=Homo sapiens OX=9606 GN=BST2 PE=1 SV=1 } \\
\text { MASTSYDYCRVPMEDGDKRCKLLGIGILV } \\
\text { LLIIVILGVPLIIFTIKANSEACRDGLRAVMEC } \\
\text { RNVTHLLQQELTEAQKGFQDVEAQAATCNHTVMALMA } \\
\text { SLDAEKAQGKKVEELEGEITLNHKLQDASAEVERL } \\
\text { RRENQVLSVRIADKKYYPSSQDSSSAAAPQLLIVLLGLSALLQ }\end{array}$ & $\begin{array}{c}6-11 \\
\mathbf{2 1 - 4 6} \\
58-62 \\
93-100 \\
144-148 \\
\mathbf{1 6 7 - 1 7 4}\end{array}$ & $\begin{array}{c}22-47 \\
92-101 \\
167-180\end{array}$ & $\begin{array}{c}22-38 \\
168-179\end{array}$ & $\begin{array}{c}\mathbf{2 5 - 4 9} \\
66-71 \\
90-96 \\
120-125 \\
141-149 \\
\mathbf{1 6 0 - 1 7 6}\end{array}$ & $\begin{array}{c}22-47 \\
93-96 \\
144-148 \\
\mathbf{1 6 7 - 1 7 8}\end{array}$ & $\begin{array}{c}\mathbf{2 2 - 4 7} \\
70-75 \\
86-104 \\
141-146 \\
\mathbf{1 6 6 - 1 8 0} \\
\text { Waltz } \\
\mathbf{2 9 - 4 7} \\
141-146 \\
\mathbf{1 6 6 - 1 8 0}\end{array}$ & $19-50$ \\
\hline
\end{tabular}

**-Results according to Amylpred 2.0 parameters; \pm -Results of individual server. Numbers in bold indicate consensus, values common in majority of servers. 
Table 8. Propensity values predicted by Chou-Fasman secondary structure prediction program for amyloid regions in tetherin protein indicating similar propensities for sheet $(\mathrm{E})$ and helix $(\mathrm{H})$ formation for region 1.

\begin{tabular}{cccc}
\hline Tetherin & H & E & T \\
\hline Region 1: 21-47 & 96.3 & 88.9 & 0.0 \\
\hline Region 2: 161-175 & 75.0 & 37.5 & 12.5 \\
\hline
\end{tabular}

Beta aggregation

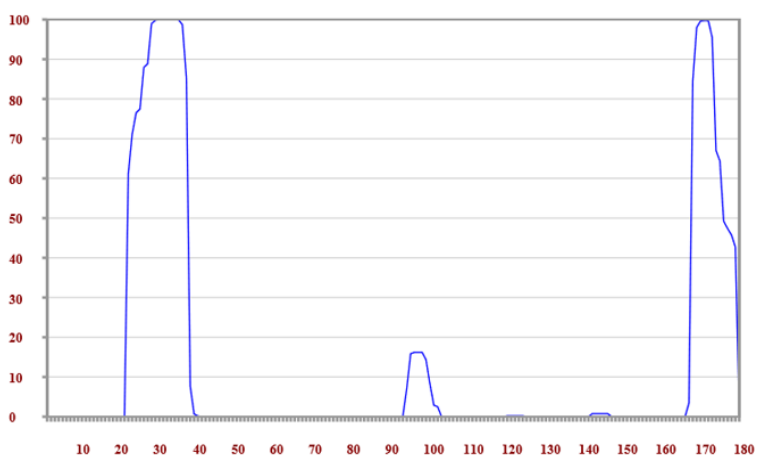

Helix aggregation

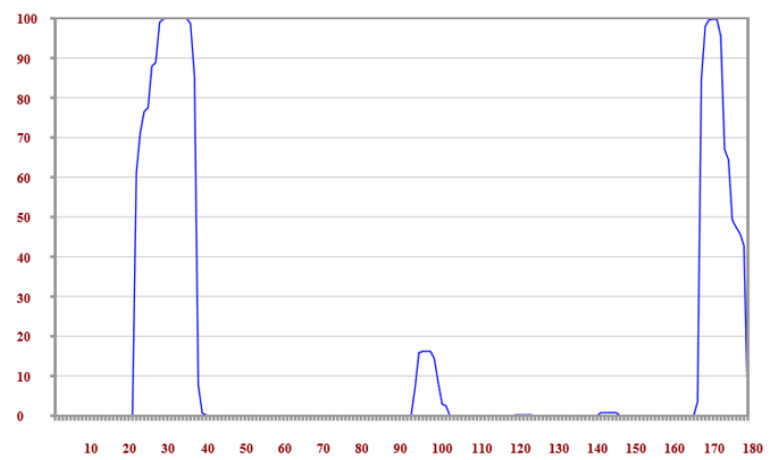

Figure 9. Beta aggregation and Helix aggregation plots generated in TANGO highlighting regions 22-38 and 168-179 to have high aggregation probability.

\section{Discussion}

Tetherin, a type II membrane protein, blocks the release of variety of enveloped viruses by retaining them on the cell surfaces, whereas HIV-1 is known to successfully overcome this blocking by crosslinking its $\mathrm{Vpu}$ protein with tetherin, making the virus resistant to the anti-viral defense mechanism [1,2]. Vpu directly interacts with the tetherin TM domain through its AxxxAxxxA motif present in the TM domain [12]. It is an established fact that HIV-1 Vpu is a highly variable protein; however, factors that contribute to Vpu sequence variability are not well defined [21].

The host immune responses may be attributed to the variability wherein polymorphism is acquired as a means of immune escape or other functional benefit [21]. Additionally, compartmentalization of HIV-1 does serve as an important aspect that would drive the sequence diversity, likely due to differential immune selection pressures, cell type-specific differences in gene expressions, and local concentrations of antiviral drugs or co-infections altering the microenvironment [20]. Compartmentalization of HIV-1 in the CNS has been very well documented and massively studied to probe the mechanisms leading to pathogenesis in HIV associated dementia (HAD) [57,58]. Case in point, the Vpu-tetherin interactions studied here have considered the HIV-1 Vpu sequences from both blood and brain compartments in a view to understand the consequence of sequence variability on binding profiles between Vpu-tetherin complexes. Our study represents the exact residues forming hydrophobic connections between HIV-1 Vpu from blood- and brain-derived isolates, and with tetherin, demonstrates the differences in the binding residues and their affinities. The consensus sequence derived from blood- and brain-derived $\mathrm{Vpu}$ sequences is also taken into account for comparison of the variations in interacting residues. Protein-protein interaction data depicts the blood-derived isolate to have maximum binding affinity with tetherin having a $\Delta \mathrm{G}$ value of $-5.0 \mathrm{kcal} / \mathrm{mol}$, while for the brain-derived and consensus $V \mathrm{pu}$ sequences, the binding affinity decreases to -3.8 and $-4.3 \mathrm{kcal} / \mathrm{mol}$, respectively. The $\mathrm{N}$-terminal region of $\mathrm{Vpu}$ that interacts with tetherin is noted to encompass a region with $\alpha$-helix/ $\beta$-sheet discordance and the same was reflected in 70ns simulation performed on brain-derived Vpu structure [23]. However, the blood-derived Vpu structure failed to show such a transition in simulations highlighting the differential behavior of Vpu from blood and brain compartments suggestively attributed to variations in the sequence. 
Specific mutations in the TM region of both Vpu or tetherin are demonstrated to be essential in rendering tetherin resistant to the Vpu counteraction [16]. From a wide range of mutations introduced in blood-derived $\mathrm{Vpu}$, as well as the tetherin TM domain, the interacting domains were verified by protein-protein docking (as indicated in Tables 5 and 6). Our study puts forth few single point as well as multiple (triple) mutations that effectively resulted in minimal hydrophobic interactions and reduced binding affinity. About 21 mutations performed in tetherin at positions L22, L23, L24, G25, I26, L29, V30, I33, I34, I36, L37, V39, P40, L41, F44, and T45, wherein single point mutations of L23 $\rightarrow$ Y, $\mathrm{L} 24 \rightarrow \mathrm{T}$, and $\mathrm{P} 40 \rightarrow \mathrm{T}$ depicted a decrease in residue pairs involved in hydrophobic interactions in the Vpu-mutant tetherin complex. Hydrophobic contacts were observed between 4 to $6 \mathrm{Vpu}$ and tetherin residues, as represented in Figure 6. The binding affinities $(\Delta \mathrm{G})$ of the L23Y, L24T, and P40T mutants were $-2.6,-2.2$, and $-2.7 \mathrm{kcal} / \mathrm{mol}$ with corresponding dissociation constants $(\mathrm{Kd})$ of $1.2 \times 10^{-3}$, $2.9 \times 10^{-3}$, and $1.9 \times 10^{-3} \mathrm{M}$, respectively. The rest of the mutations showed a reduction in binding affinity between $\mathrm{Vpu}$ and mutant tetherin molecules. There were about 44 possible complexes with all possible conformations between the two proteins, indicating at least few interacting residues in each conformation. The reported T45I single mutation that causes tetherin to become resistant to Vpu-mediated depletion [16] was also tested in our study. However, the protein-protein complex was found to be associated with few hydrophobic residue interactions and binding affinity of $-2.5 \mathrm{kcal} / \mathrm{mol}$. The combined/multiple mutations at $\{$ L22S, F44Y, L37I\} and \{L23T, L37T, T45I\} showed minimal hydrophobic connections and low binding affinities in Vpu-tetherin complexes with binding affinities $(\Delta \mathrm{G})$ and dissociation constant $(\mathrm{Kd})$ of $-2.2 \mathrm{kcal} / \mathrm{mol}$ and $1.8 \times 10^{-2} \mathrm{M}$ and $-2.9 \mathrm{kcal} / \mathrm{mol}$ and $2.3 \times 10^{-2}$ $\mathrm{M}$, respectively. Interestingly, these sets of residues are observed to be interacting in most of the mutant tetherin-Vpu complexes. A computer-assisted structural modeling and mutagenesis study predicted that an alignment of four amino acid residues such as I34, L37, L41, and T45 on the same helical face in the TM domain of tetherin is important for the Vpu-mediated antagonism of human tetherin [14]. Hence, the mutations in these residues may prove crucial in breaking the contacts and decreasing the binding affinity. Out of the 22 mutations performed in blood-derived Vpu at S3, Q5, L7, A8, V10, A11, $\mathrm{V} 14, \mathrm{~A} 15, \mathrm{I17}, \mathrm{I18}, \mathrm{A} 19, \mathrm{~W} 23, \mathrm{I25}, \mathrm{F} 27, \mathrm{R} 31$, and K32 single point mutations of A19 $\rightarrow \mathrm{H}$ and $\mathrm{W} 23 \rightarrow \mathrm{Y}$ affected the hydrophobic interactions in mutant $\mathrm{Vpu}$ and tetherin, while combined mutations of \{V10K, A11L, A19T\}, \{V14T, I18T, I26S\}, and \{A11T, V14L, A15T\} showed minimal hydrophobic connections and low binding affinities in the docked complexes, as represented in Figure 7. The docked complexes of $\mathrm{A} 19 \mathrm{H}$ and $\mathrm{W} 23 \mathrm{Y}$ Vpu mutants possessed binding affinities $(\Delta \mathrm{G})$ as -2.5 and $-2.1 \mathrm{kcal} / \mathrm{mol}$ and $\mathrm{Kd}$ of $2.9 \times 10^{-3}$ and $2.1 \times 10^{-3} \mathrm{M}$, while the complexes with triple mutations $\{\mathrm{V} 10 \mathrm{~K}, \mathrm{~A} 11 \mathrm{~L}, \mathrm{~A} 19 \mathrm{~T}\}$, \{V14T, I18T, I26S\}, and $\{$ A11T, V14L, A15T\} had binding affinities $(\Delta \mathrm{G})$ of $-2.6,-2.7$, and $-2.3 \mathrm{kcal} / \mathrm{mol}$ and the corresponding Kds of $2.1 \times 10^{-5}, 2.2 \times 10^{-4}$, and $2.8 \times 10^{-2} \mathrm{M}$, respectively. The amino acids $\mathrm{A} 11, \mathrm{~V} 13, \mathrm{~V} 14, \mathrm{~A} 15, \mathrm{I} 17, \mathrm{I} 25$, and I28 of Vpu are frequently observed in the interactions of mutant Vpu-tetherin complexes. A study associating the biological activity of the TM hetero-dimers of HIV-1 $\mathrm{Vpu}$ and its host factors with computationally-derived structural features suggests the importance of the tilt in Vpu's alanine rim Ala-8/11/15/19 [17]. This study implicates the reduction in parallel alignment to correlate with low activity. On similar lines, these alanine residues are mutated in our study and, as mutation in A15F is observed to have higher downregulation activity than A19F in a study by Li et al., A15T and A19H mutation in our study resulted in reduced hydrophobic interactions, suggesting the absence of a strong connection must be impacting the tilt of the Alanine rim. In a futuristic view, tetherin or Vpu binding agents might prove essential in protecting tetherin from viral encoded counteractions and enhance its anti-viral properties. Interestingly, a study has also reported that a cholesterol-binding compound, Amphotericin B methyl ester, inhibits the HIV-1 assembly and releases by interfering with the anti-CD317/BST-2/tetherin function of Vpu [59].

Another aspect of this study was the investigation of aggregating potential of tetherin. The various amyloid predicting programs suggested the presence of amyloidogenic stretch at position 21-47 and 161-176 in tetherin. The region 21-47 is a TM helical region which is also predicted to encompass a $\alpha$-helix $/ \beta$-sheet discordant region with residues having higher propensities for helix, as well as sheet. 
It is a well-known fact that the neurodegenerative diseases are characterized by protein aggregation and deposition of insoluble amyloid fibrils in the brain [60]. Also, a presence of discordant region is quite a common property in these amyloidogenic proteins [61]. Moreover, these features are also shared in the development of HAD/HIV-associated neurocognitive disorders (HAND) $[62,63]$.

Numerous studies have presented findings of amyloid aggregates in the brains of HIV-infected patients, either due to accumulation of $\beta$-amyloid precursors or viral-derived aggregating proteins [6469]. However, work on recognizing the exact mechanisms leading to pathogenesis in HAD and role of amyloids in its enhancement is still underway. Functionally, HIV-1 Vpu is involved in directing the ubiquitination and degradation of tetherin by interacting with its TM region, wherein Vpu acts as an adapter molecule linking tetherin to the cellular ubiquitination machinery via $\beta \operatorname{TrCP}[10]$. It is prospective that, during this degradation process, tetherin could possibly aggregate and evade the activity of ubiquitin-proteasome machinery. Although almost all of the proteins encoded by the human genome can be efficiently removed from the cell when misfolded, a number of polypeptides produced from post-translational conjugation, such as hyperphosphorylated tau in Alzheimer's disease (AD), or due to endoproteolytic cleavage, such as amyloid $\beta$ peptides, tend to be rapidly aggregated into oligomers enriched in $\beta$-sheet and escape the regular degradation process [70-73]. Such oligomers are at least partially resistant to all known proteolytic pathways, further leading to inclusion bodies or extracellular plaques having highly ordered $\beta$-sheet fibrils [70]. On these lines, the predicted amyloidogenic potential of tetherin needs to be further studied to affirm this possibility and investigate its involvement in progress of dementia.

\section{Conclusions}

The observation of best binding affinity between blood-derived Vpu and tetherin, in comparison to brain-derived and consensus HIV-1 Vpu proteins, reflects the effect of sequence variations in compartmentalized isolates on their binding potential to tetherin. The computational protein-protein interaction of tetherin and Vpu mutant complexes highlights the consistent hydrophobic interaction of key residues A11, V13, V14, A15, I17, I25, and I28 of Vpu and L22, L23, L24, I33, V30, I36, L37, P40, I43, and F44 of tetherin in the majority of the complexes, despite the various mutations, suggesting their essential involvement in binding. The extensive mutational analysis further charts out that the selective single point and/or triple mutations in the residues at positions L22, L23, L24, L37, P40, and F44 of tetherin and V10, A11, V14, A15, I18, A19, W23, and I26 of Vpu results in a decrease in hydrophobic interactions and reduced binding affinities. Additionally, amyloidogenecity prediction of tetherin has revealed its possible aggregation potential that needs to be further explored for its underlying contribution in dementia progression. Our study provides a basic approach to investigate the interacting residues and possible mutations, as well as to understand the connectivity aspects between HIV-1 Vpu and tetherin.

Author Contributions: For research articles with several authors, a short paragraph specifying their individual contributions must be provided. The following statements should be used conceptualization, P.S. and S.B; methodology, P.S. and S.B; software, P.S. and U.S.; validation, P.S. and S.B; formal analysis, P.S. and S.B; investigation, P.S., U.S. and S.B; resources, P.S., U.S. and S.B; data curation, P.S. and S.B; writing-original draft preparation, P.S., U.S., and S.B; writing—review and editing, P.S. and S.B; visualization, P.S. and U.S.; supervision, S.B; project administration, P.S. and S.B; funding acquisition, S.B.

Funding: The corresponding author S.B. acknowledges the grant (No. VGST/GRD-533/2016-17/241) received from Karnataka Science and Technology Promotion Society (KSTePS), India, for supporting the Centre for Interactive Biomolecular 3D-literacy (C-in-3D) under the VGST scheme-Centres of Innovative Science, Engineering and Education (CISEE) for the year 2018-19.

Conflicts of Interest: The authors declare no conflict of interest.

\section{References}

1. Le Tortorec, A.; Willey, S.; Neil, S.J. Antiviral Inhibition of Enveloped Virus Release by Tetherin/BST-2: Action and Counteraction. Viruses 2011, 3, 520-540. [CrossRef] [PubMed] 
2. Evans, D.T.; Serra-Moreno, R.; Singh, R.K.; Guatelli, J.C. BST-2/tetherin: A new component of the innate immune response to enveloped viruses. Trends Microbiol. 2010, 18, 388-396. [CrossRef] [PubMed]

3. Kupzig, S.; Korolchuk, V.; Rollason, R.; Sugden, A.; Wilde, A.; Banting, G. Bst-2/HM1.24 Is a Raft-Associated Apical Membrane Protein with an Unusual Topology. Traffic 2003, 4, 694-709. [CrossRef] [PubMed]

4. Arias, J.F.; Iwabu, Y.; Tokunaga, K. Structural Basis for the Antiviral Activity of BST-2/Tetherin and Its Viral Antagonism. Front. Microbiol. 2011, 2, 250. [CrossRef] [PubMed]

5. Bieniasz, P.D. The cell biology of HIV-1 virion genesis. Cell Host Microbe 2009, 5, 550-558. [CrossRef] [PubMed]

6. Neil, S.J.; Zang, T.; Bieniasz, P.D. Tetherin inhibits retrovirus release and is antagonized by HIV-1 Vpu. Nature 2008, 451, 425-430. [CrossRef]

7. Nomaguchi, M.; Fujita, M.; Adachi, A. Role of HIV-1 Vpu protein for virus spread and pathogenesis. Microbes Infect. 2008, 10, 960-967. [CrossRef]

8. Mangeat, B.; Gers-Huber, G.; Lehmann, M.; Zufferey, M.; Luban, J.; Piguet, V. HIV-1 Vpu Neutralizes the Antiviral Factor Tetherin/BST-2 by Binding It and Directing Its Beta-TrCP2-Dependent Degradation. PLoS Pathog. 2009, 5, e1000574. [CrossRef]

9. Iwabu, Y.; Fujita, H.; Kinomoto, M.; Kaneko, K.; Ishizaka, Y.; Tanaka, Y.; Tokunaga, K. HIV-1 Accessory Protein Vpu Internalizes Cell-surface BST-2/Tetherin through Transmembrane Interactions Leading to Lysosomes. J. Biol. Chem. 2009, 284, 35060-35072. [CrossRef]

10. Douglas, J.L.; Viswanathan, K.; McCarroll, M.N.; Gustin, J.K.; Fruh, K.; Moses, A.V. Vpu Directs the Degradation of the Human Immunodeficiency Virus Restriction Factor BST-2/Tetherin via a TrCP-Dependent Mechanism. J. Virol. 2009, 83, 7931-7947. [CrossRef]

11. McNatt, M.W.; Zang, T.; Bieniasz, P.D. Vpu binds directly to tetherin and displaces it from nascent virions. PLoS Pathog. 2013, 9, e1003299. [CrossRef] [PubMed]

12. Skasko, M.; Wang, Y.; Tian, Y.; Tokarev, A.; Munguia, J.; Ruiz, A.; Guatelli, J. HIV-1 Vpu Protein Antagonizes Innate Restriction Factor BST-2 via Lipid-embedded Helix-Helix Interactions. J. Biol. Chem. 2011, 287, 58-67. [CrossRef]

13. Tokarev, A.; Skasko, M.; Fitzpatrick, K.; Guatelli, J. Antiviral Activity of the Interferon-Induced Cellular Protein BST-2/Tetherin. AIDS Res. Hum. Retrovir. 2009, 25, 1197-1210. [CrossRef] [PubMed]

14. Kobayashi, T.; Ode, H.; Yoshida, T.; Sato, K.; Gee, P.; Yamamoto, S.P.; Koyanagi, Y. Identification of Amino Acids in the Human Tetherin Transmembrane Domain Responsible for HIV-1 Vpu Interaction and Susceptibility. J. Virol. 2010, 85, 932-945. [CrossRef] [PubMed]

15. Vigan, R.; Neil, S.J. Determinants of Tetherin Antagonism in the Transmembrane Domain of the Human Immunodeficiency Virus Type 1 Vpu Protein. J. Virol. 2010, 84, 12958-12970. [CrossRef] [PubMed]

16. Gupta, R.K.; Hué, S.; Schaller, T.; Verschoor, E.; Pillay, D.; Towers, G.J. Mutation of a Single Residue Renders Human Tetherin Resistant to HIV-1 Vpu-Mediated Depletion. PLoS Pathog. 2009, 5, e1000443. [CrossRef] [PubMed]

17. Pang, X.; Hu, S.; Li, J.; Xu, F.; Mei, S.; Zhou, J.; Cen, S.; Jin, Q.; Guo, F. Identification of novel key amino acids at the interface of the transmembrane domains of human BST-2 and HIV-1 Vpu. Retrovirology 2013, 10, 84. [CrossRef] [PubMed]

18. Li, L.; Fischer, W.B. Correlation of biological activity with computationally derived structural features from transmembrane hetero-dimers of HIV-1 Vpu with host factors. Biochim. Biophys. Acta Biomembr. 2014, 1838, 1104-1112. [CrossRef] [PubMed]

19. Skasko, M.; Tokarev, A.; Chen, C.; Fischer, W.B.; Pillai, S.K.; Guatelli, J. BST-2 is rapidly down-regulated from the cell surface by the HIV-1 protein Vpu: Evidence for a post-ER mechanism of Vpu-action. Virology 2011, 411, 65-77. [CrossRef]

20. Blackard, J.T. HIV Compartmentalization: A Review on a Clinically Important Phenomenon. Curr. HIV Res. 2012, 10, 133-142. [CrossRef]

21. Hasan, Z. Role of host immune responses in sequence variability of HIV-1 Vpu. World J. Immunol. 2014, 4, 107. [CrossRef]

22. Balaji, S.; Sneha, P.; Rama, M.; Shapshak, P. Global Protein Sequence Variation in HIV-1-B Isolates Derived from Human Blood and Brain. Glob. Virol. Identif. Investig. Viral Dis. 2015, 1, 613-666. [CrossRef] 
23. Sneha, P.; Panda, P.K.; Gharemirshamlu, F.R.; Bamdad, K.; Balaji, S. Structural discordance in HIV-1 Vpu from brain isolate alarms amyloid fibril forming behavior-a computational perspective. J. Theor. Biol. 2018, 451, 35-45. [CrossRef] [PubMed]

24. Berman, H.M. The Protein Data Bank. Nucleic Acids Res. 2000, 28, 235-242. [CrossRef] [PubMed]

25. Sievers, F.M.; Wilm, A.; Dineen, D.; Gibson, T.J.; Karplus, K.; Li, W.; Higgins, D.G. Fast, scalable generation of high-quality protein multiple sequence alignments using Clustal Omega. Mol. Syst. Biol. 2014, 7, 539. [CrossRef] [PubMed]

26. Chojnacki, S.; Cowley, A.; Lee, J.; Foix, A.; Lopez, R. Programmatic access to bioinformatics tools from EMBL-EBI update: 2017. Nucleic Acids Res. 2017, 45, W550-W553. [CrossRef] [PubMed]

27. Altschul, S.F.; Gish, W.; Miller, W.; Myers, E.W.; Lipman, D.J. Basic local alignment search tool. J. Mol. Biol. 1990, 215, 403-410. [CrossRef]

28. Schwede, T. Swiss-Model: An automated protein homology-modeling server. Nucleic Acids Res. 2003, 31, 3381-3385. [CrossRef] [PubMed]

29. Wiederstein, M.; Sippl, M.J. ProSA-web: Interactive web service for the recognition of errors in three-dimensional structures of proteins. Nucleic Acids Res. 2007, 35, W407-W410. [CrossRef] [PubMed]

30. Sippl, M.J. Recognition of errors in three-dimensional structures of proteins. Proteins Struct. Funct. Genet. 1993, 17, 355-362. [CrossRef] [PubMed]

31. Ritchie, D.W.; Kemp, G.J. Protein docking using spherical polar Fourier correlations. Proteins Struct. Funct. Genet. 2000, 39, 178. [CrossRef]

32. Ritchie, D.W.; Kozakov, D.; Vajda, S. Accelerating and focusing protein-protein docking correlations using multi-dimensional rotational FFT generating functions. Bioinformatics 2008, 24, 1865-1873. [CrossRef] [PubMed]

33. Vangone, A.; Bonvin, A.M. Contacts-based prediction of binding affinity in protein-protein complexes. ELife 2015, 4, e07454. [CrossRef]

34. Xue, L.C.; Rodrigues, J.P.; Kastritis, P.L.; Bonvin, A.M.; Vangone, A. Prodigy: A web server for predicting the binding affinity of protein-protein complexes. Bioinformatics 2016, 32, 3676-3678. [CrossRef] [PubMed]

35. DeLano, W.L. The PyMOL Molecular Graphics System. Available online: http://pymol.org (accessed on 19 May 2019).

36. Guex, N.; Peitsch, M.C. SWISS-MODEL and the Swiss-Pdb Viewer: An environment for comparative protein modeling. Electrophoresis 1997, 18, 2714-2723. [CrossRef] [PubMed]

37. Tina, K.G.; Bhadra, R.; Srinivasan, N. PIC: Protein Interactions Calculator. Nucleic Acids Res. 2007, 35, W473-W476. [CrossRef] [PubMed]

38. Capriotti, E.; Fariselli, P.; Casadio, R. I-Mutant2.0: Predicting stability changes upon mutation from the protein sequence or structure. Nucleic Acids Res. 2005, 33, W306-W310. [CrossRef] [PubMed]

39. Kumar, P.; Henikoff, S.; Ng, P.C. Predicting the effects of coding non-synonymous variants on protein function using the SIFT algorithm. Nat. Protoc. 2009, 4, 1073-1081. [CrossRef] [PubMed]

40. Pettersen, E.F.; Goddard, T.D.; Huang, C.C.; Couch, G.S.; Greenblatt, D.M.; Meng, E.C.; Ferrin, T.E. UCSF Chimera? A visualization system for exploratory research and analysis. J. Comput. Chem. 2004, 25, 1605-1612. [CrossRef] [PubMed]

41. Monsellier, E.; Chiti, F. Prevention of amyloid-like aggregation as a driving force of protein evolution. EMBO Rep. 2007, 8, 737-742. [CrossRef]

42. Chiti, F.; Stefani, M.; Taddei, N.; Ramponi, G.; Dobson, C.M. Rationalization of the effects of mutations on peptide andprotein aggregation rates. Nature 2003, 424, 805-808. [CrossRef] [PubMed]

43. Sunde, M.; Serpell, L.C.; Bartlam, M.; Fraser, P.E.; Pepys, M.B.; Blake, C.C. Common core structure of amyloid fibrils by synchrotron X-ray diffraction. J. Mol. Biol. 1997, 273, 729-739. [CrossRef] [PubMed]

44. Garbuzynskiy, S.O.; Lobanov, M.Y.; Galzitskaya, O.V. FoldAmyloid: A method of prediction of amyloidogenic regions from protein sequence. Bioinformatics 2009, 26, 326-332. [CrossRef] [PubMed]

45. Conchillo-Solé, O.; De Groot, N.S.; Avilés, F.X.; Vendrell, J.; Daura, X.; Ventura, S. AGGRESCAN: A server for the prediction and evaluation of "hot spots" of aggregation in polypeptides. BMC Bioinf. 2007, 8, 65. [CrossRef]

46. Fernandez-Escamilla, A.; Rousseau, F.; Schymkowitz, J.; Serrano, L. Prediction of sequence-dependent and mutational effects on the aggregation of peptides and proteins. Nat. Biotechnol. 2004, 22, 1302-1306. [CrossRef] [PubMed] 
47. Maurer-Stroh, S.; Debulpaep, M.; Kuemmerer, N.; De la Paz, M.L.; Martins, I.C.; Reumers, J.; Rousseau, F. Exploring the sequence determinants of amyloid structure using position-specific scoring matrices. Nat. Methods 2010, 7, 237-242. [CrossRef] [PubMed]

48. Emily, M.; Talvas, A.; Delamarche, C. MetAmyl: A METa-Predictor for AMYLoid Proteins. PLoS ONE 2013, 8, e79722. [CrossRef] [PubMed]

49. Tsolis, A.C.; Papandreou, N.C.; Iconomidou, V.A.; Hamodrakas, S.J. A Consensus Method for the Prediction of 'Aggregation-Prone' Peptides in Globular Proteins. PLoS ONE 2013, 8, e54175. [CrossRef] [PubMed]

50. Walsh, I.; Seno, F.; Tosatto, S.C.; Trovato, A. PASTA 2.0: An improved server for protein aggregation prediction. Nucleic Acids Res. 2014, 42, W301-W307. [CrossRef] [PubMed]

51. Lopez de la Paz, M.; Serrano, L. Sequence determinants of amyloid fibril formation. Proc. Natl. Acad. Sci. USA 2013, 101, 87-92. [CrossRef] [PubMed]

52. Zibaee, S.; Makin, O.S.; Goedert, M.; Serpell, L.C. A simple algorithm locates $\beta$-strands in the amyloid fibril core of $\alpha$-synuclein, $A \beta$, and tau using the amino acid sequence alone. Protein Sci. 2007, 16, 906-918. [CrossRef] [PubMed]

53. Zhang, Z.; Chen, H.; Lai, L. Identification of amyloid fibril-forming segments based on structure and residue-based statistical potential. Bioinformatics 2007, 23, 2218-2225. [CrossRef] [PubMed]

54. Chou, P.Y.; Fasman, G.D. Prediction of protein conformation. Biochemistry 1974, 13, 222-245. [CrossRef] [PubMed]

55. Ha, J.; Loh, S.N. Protein Conformational Switches: From Nature to Design. Chem. Eur. J. 2012, 18, 7984-7999. [CrossRef] [PubMed]

56. Guo, F.; Liang, C. Transmembrane Interactions of HIV-1 Vpu and Tetherin. Curr. HIV Res. 2012, 10, $292-297$. [CrossRef] [PubMed]

57. Burkala, E.J.; He, J.; West, J.T.; Wood, C.; Petito, C.K. Compartmentalization of HIV-1 in the central nervous system: Role of the choroid plexus. AIDS 2005, 19, 675-684. [CrossRef] [PubMed]

58. Ghafouri, M.; Amini, S.; Khalili, K.; Sawaya, B.E. HIV-1 associated dementia: Symptoms and causes. Retrovirology 2006, 3, 28. [CrossRef]

59. Waheed, A.A.; Ablan, S.D.; Soheilian, F.; Nagashima, K.; Ono, A.; Schaffner, C.P.; Freed, E.O. Inhibition of Human Immunodeficiency Virus Type 1 Assembly and Release by the Cholesterol-Binding Compound Amphotericin B Methyl Ester: Evidence for Vpu Dependence. J. Virol. 2008, 82, 9776-9781. [CrossRef]

60. Ross, C.A.; Poirier, M.A. Protein aggregation and neurodegenerative disease. Nat. Med. 2004, 10, S10-S17. [CrossRef]

61. Kallberg, Y.; Gustafsson, M.; Persson, B.; Thyberg, J.; Johansson, J. Prediction of amyloid fibril forming proteins. J. Biol. Chem. 2001, 276, 12945-12950. [CrossRef]

62. Brew, B.J.; Crowe, S.M.; Landay, A.; Cysique, L.A.; Guillemin, G. Neurodegeneration and Ageing in the HAART Era. J. Neuroimmune Pharmacol. 2008, 4, 163-174. [CrossRef] [PubMed]

63. Khanlou, N.; Khanlou, N.; Moore, D.J.; Khanlou, N.; Moore, D.J.; Chana, G. The HNRC Group. Increased frequency of $\alpha$-synuclein in the substantia nigra in human immunodeficiency virus infection. J. Neurovirol. 2009, 15, 131-138. [CrossRef] [PubMed]

64. Green, D.A.; Masliah, E.; Vinters, H.V.; Beizai, P.; Moore, D.J.; Achim, C.L. Brain deposition of beta-amyloid is a common pathologic feature in HIV positive patients. AIDS 2005, 19, 407-411. [CrossRef] [PubMed]

65. András, I.E.; Toborek, M. Amyloid beta accumulation in HIV-1-infected brain: The role of the blood brain barrier. IUBMB Life 2012, 65, 43-49. [CrossRef]

66. Rempel, H.C.; Pulliam, L. HIV-1 Tat inhibits neprilysin and elevates amyloid $\beta$. AIDS 2005, 19, 127-135. [CrossRef] [PubMed]

67. Chen, X.; Hui, L.; Geiger, N.H.; Haughey, N.J.; Geiger, J.D. Endolysosome in-volvement in HIV-1 transactivator protein-induced neuronal amyloid beta pro-duction. Neurobiology 2013, 34, 2370-2378.

68. Aksenov, M.Y.; Aksenova, M.V.; Mactutus, C.F.; Booze, R.M. HIV-1 protein-me-diated amyloidogenesis in rat hippocampal cell cultures. Neurosci. Lett. 2010, 475, 174-178. [CrossRef] [PubMed]

69. Wojtowicz, W.M.; Farzan, M.; Joyal, J.L.; Carter, K.; Babcock, G.J.; Israel, D.I.; So-droski, J.; Mirzabekov, T. Stimulation of enveloped virus infection by $\beta$-amyloid fibrils. J. Biol. Chem. 2002, 277, 35019-35024. [CrossRef]

70. Kopito, R.R. Aggresomes, inclusion bodies and protein aggregation. Trends Cell Biol. 2000, 10, 524-530. [CrossRef] 
71. Hardy, J. The Amyloid Hypothesis of Alzheimer's Disease: Progress and Problems on the Road to Therapeutics. Science 2002, 297, 353-356. [CrossRef]

72. Huang, Y.; Mucke, L. Alzheimer Mechanisms and Therapeutic Strategies. Cell 2012, 148, 1204-1222. [CrossRef] [PubMed]

73. Ciechanover, A.; Kwon, Y.T. Degradation of misfolded proteins in neurodegenerative diseases: Therapeutic targets and strategies. Exp. Mol. Med. 2015, 47, e147. [CrossRef] [PubMed] 